Collection SFN 9 (2008) 87-110

(C) EDP Sciences, Les Ulis

DOI: $10.1051 / \mathrm{sfn}: 2008007$

\title{
Structures magnétiques, densités d'aimantation et facteurs de forme magnétiques mesurés par diffraction de neutrons polarisés
}

\section{J. Schweizer}

\section{CEA-Grenoble, INAC/SPSMSIMDN, 17 rue des Martyrs, 38054 Grenoble, France}

La diffraction de neutrons est un outil exceptionnel pour déterminer les structures magnétiques, c'est à dire l'arrangement des moments des différents atomes magnétiques à l'intérieur du solide. A une échelle plus petite, les neutrons peuvent aussi révéler, dans tous leurs détails, les distributions de la densité d'aimantation M(r) autour des noyaux. Cette aimantation est la somme de l'aimantation due aux spins et de l'aimantation due aux orbites des électrons non appariés. Elle porte des informations essentielles concernant certains aspects de la structure électronique du cristal, comme par exemple la liaison chimique, la délocalisation des fonctions d'onde magnétiques, la polarisation de spin ou l'interaction magnétiques entre molécules voisines.

La détermination précise de ces densités d'aimantation requiert une très bonne connaissance des facteurs de structure magnétiques, même des plus faibles, ce qui implique des mesures de diffraction avec des neutrons polarisés.

\section{LA MESURE PRÉCISE DES AMPLITUDES MAGNÉTIQUES AVEC DES NEUTRONS POLARISÉS}

Nous allons examiner dans cette section comment, dans le but de déterminer les densités de spin (ou d'aimantation), on peut utiliser les neutrons polarisés pour mesurer les amplitudes magnétiques avec précision. Nous allons considérer successivement le principe de la méthode et sa mise en œuvre.

\subsection{Le principe de la diffraction de neutrons polarisés}

Les facteurs de structure magnétiques $\mathrm{F}_{\mathrm{M}}$ sont les coefficients de Fourier de la fonction périodique $\mathrm{M}(\mathbf{r})$ :

$$
\mathrm{F}_{\mathrm{M}}(\mathbf{Q})=\iiint \mathrm{M}(\mathbf{r}) \mathrm{e}^{\mathrm{i} \mathbf{Q} \mathbf{r}} .
$$

Pour des composés ferro ou ferrimagnétiques, mais aussi pour des paramagnétiques ou des antiferromagnétiques pour lesquels une composante ferro a été induite par un champ magnétique appliqué, des réflexions magnétiques apparaissent là où se trouvent déjà les réflexions nucléaires. Les neutrons polarisés prennent avantage du couplage entre les amplitudes qui existent pour ces réflexions de Bragg communes (vecteur de propagation $\mathbf{k}=0$ ). Pour des neutrons non polarisés l'intensité de ces réflexions est donné par:

$$
\mathrm{I}=\left|\mathrm{F}_{\mathrm{N}}\right|^{2}+\left|\mathrm{F}_{\mathrm{M}}\right|^{2}
$$

où $F_{N}$ et $F_{M}$ sont les facteurs de structure nucléaire et magnétique. Pour les petits $F_{M}$ la sensibilité est énormément améliorée par l'utilisation de neutrons polarisés dans la mesure où l'intensité des réflexions de Bragg dépend maintenant de la polarisation du faisceau incident, de la façon suivante :

$$
\mathrm{I}^{ \pm}=\left(\mathrm{F}_{\mathrm{N}} \pm \mathrm{F}_{\mathrm{M}}\right)^{2} .
$$

Pour illustrer la sensibilité de la diffraction de neutrons polarisés dans le cas de petites amplitudes magnétiques, prenons un exemple où $\mathrm{F}_{\mathrm{M}}=0.1 \mathrm{~F}_{\mathrm{N}}$. Des neutrons non polarisés seraient diffractés avec 
une intensité :

$$
\mathrm{I}=\mathrm{F}_{\mathrm{N}}^{2}+\left(0.1 \mathrm{~F}_{\mathrm{N}}\right)^{2}=1.01 \mathrm{~F}_{\mathrm{N}}^{2}
$$

tandis que des neutrons polarisés donneraient, pour les deux directions possibles de la polarisation :

$$
\begin{aligned}
& \mathrm{I}^{+}=\left(\mathrm{F}_{\mathrm{N}}+0.1 \mathrm{~F}_{\mathrm{N}}\right)^{2}=1.21 \mathrm{~F}_{\mathrm{N}}^{2} \\
& \mathrm{I}^{-}=\left(\mathrm{F}_{\mathrm{N}}-0.1 \mathrm{~F}_{\mathrm{N}}\right)^{2}=0.81 \mathrm{~F}_{\mathrm{N}}^{2} .
\end{aligned}
$$

L'augmentation de la sensibilité est gigantesque.

\subsection{Réalisation d'une expérience de diffraction de neutrons}

Comme une expérience de diffraction de neutrons polarisés exige une bonne connaissance de la structure nucléaire du composé étudié, la première étape dans une recherche de densité d'aimantation (de spin) est une collecte d'intensités à basse température avec des neutrons non polarisés. Cette collecte permet l'affinement de la structure cristalline : la position des atomes (y compris les atomes légers comme l'hydrogène), les paramètres d'agitation thermique, les paramètres d'extinction, dans les mêmes conditions que celles de l'expérience avec les neutrons polarisés.

Le cristal est ensuite monté dans le cryoaimant du diffractomètre à neutrons polarisés. Le champ appliqué est vertical (parallèle à l'axe z), et la polarisation du faisceau incident lui est parallèle. Le cristal ne peut tourner qu'autour de cet axe vertical $\mathrm{Oz}$, mais le détecteur du diffractomètre peut se déplacer au dessus et au dessous du plan équatorial pour aller chercher les réflexions diffractées et les mesurer. Le faisceau incident est polarisé et monochromatique, grâce à un monochromateur qui est en même temps un polariseur. La polarisation est renversée à la demande, par un flipper inséré entre le monochromateur et l'échantillon (voir figure 1).

Pour chaque réflexion de Bragg on mesure les intensités $\mathrm{I}^{+}$et $\mathrm{I}^{-}$pour les deux états de polarisation "up" et "down" du faisceau incident. En fait, ce qui est réellement mesuré, c'est le rapport de

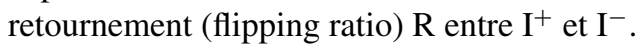

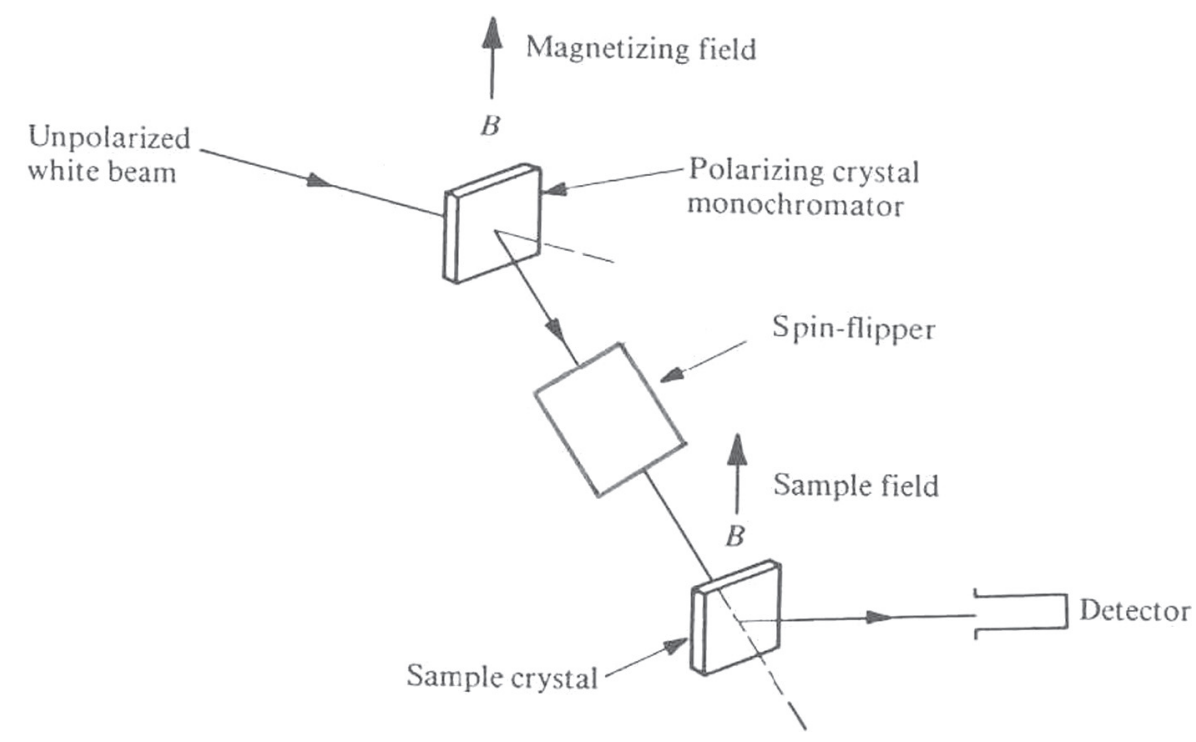

Figure 1. Principe d'une expérience de diffraction avec des neutrons polarisés. 


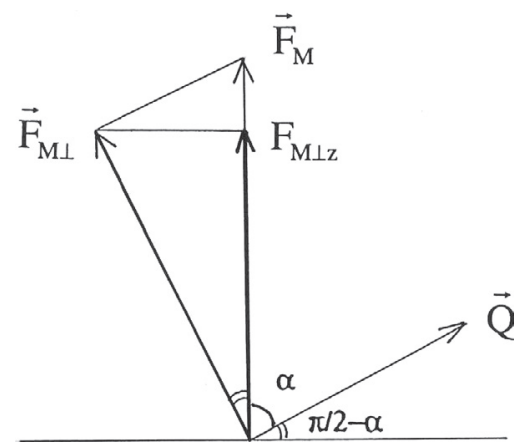

Figure 2. Relations entre les vecteurs $\mathbf{F}_{\mathrm{M}}, \mathbf{F}_{\mathrm{M} \perp}$ et $\mathbf{F}_{\mathrm{M} \perp z}$ avec un vecteur de diffusion $\mathbf{Q}$ faisant un angle $\alpha$ avec l'axe Oz.

Il faut rappeler ici, d'une part que le facteur de structure magnétique est un vecteur $\mathbf{F}_{\mathbf{M}}$, a priori complexe, avec une partie réelle $\mathbf{F}_{\mathrm{M}}^{\prime \prime}$ et une partie imaginaire $\mathbf{F}_{\mathrm{M}}^{\prime \prime}$, et d'autre part que dans le processus de diffusion, la partie active du facteur de structure magnétique est sa projection $\mathbf{F}_{\mathbf{M} \perp}$ sur le plan perpendiculaire au vecteur de diffusion $\mathbf{Q}$. Avec ces considérations, l'expression correcte pour les intensités des réflexions avec des neutrons polarisés est :

$$
\mathrm{I}^{ \pm}=\left|\mathrm{F}_{\mathrm{N}}\right|^{2} \pm\left(\mathrm{F}_{\mathrm{N}} \mathrm{F}_{\mathrm{M} \perp \mathrm{z}}^{*}+\mathrm{F}_{\mathrm{N}}^{*} \mathrm{~F}_{\mathrm{M} \perp \mathrm{z}}\right)+\left|\mathrm{F}_{\mathrm{M} \perp}^{2}\right|
$$

et le rapport de flipping s'écrit :

$$
\mathrm{R}=\frac{\mathrm{I}^{+}}{\mathrm{I}^{-}}=\frac{\left|\mathrm{F}_{\mathrm{N}}\right|^{2}+\left(\mathrm{F}_{\mathrm{N}} \mathrm{F}_{\mathrm{M} \perp \mathrm{z}}^{*}+\mathrm{F}_{\mathrm{N}}^{*} \mathrm{~F}_{\mathrm{M} \perp \mathrm{z}}\right)+\mid \mathrm{F}_{\left.\mathrm{M} \perp\right|^{2}}}{\left|\mathrm{~F}_{\mathrm{N}}\right|^{2}+\left(\mathrm{F}_{\mathrm{N}} \mathrm{F}_{\mathrm{M} \perp \mathrm{z}}^{*}+\mathrm{F}_{\mathrm{N}}^{*} \mathrm{~F}_{\mathrm{M} \perp \mathrm{z}}\right)+\left|\mathrm{F}_{\mathrm{M} \perp}\right|^{2}} .
$$

Cette relation (5), qui relie les facteurs de structure magnétiques à déterminer, aux facteurs de structure nucléaires déjà déterminés et aux rapports de flipping mesurés, est difficile à traiter dans la mesure où elle implique une équation avec trois quantités inconnues: $\mathrm{F}_{\mathrm{M} \perp \mathrm{z}}^{\prime}, \mathrm{F}_{\mathrm{M} \perp \mathrm{z}}^{\prime \prime}$ et $\mid \mathbf{F}_{\left.\mathbf{M} \perp\right|^{2}}$.

Cependant, dans beaucoup de cas, la situation est simplifiée car deux conditions sont remplies :

1) la structure cristalline est centro-symétrique, ce qui implique que les deux facteurs de structure $\mathbf{F}_{\mathbf{N}}$ et

$\mathbf{F}_{\mathbf{M}}$ sont réels

2) l'aimantation des atomes est parfaitement alignée par le champ magnétique, c'est à dire parallèle

à la direction $\mathrm{Oz}$. Les valeurs de $\mathrm{F}_{\mathrm{M} \perp \mathrm{z}}$ et $\left|\mathrm{F}_{\mathrm{M} \perp}\right|^{2}$ s'expriment alors simplement en fonction de l'angle $\alpha$, l'angle entre le vecteur de diffusion $\mathbf{Q}$ et la direction $\mathrm{Oz}$ (voir figure 2).

$$
\begin{gathered}
F_{M \perp z}=F_{M} \sin \alpha \\
\left|F_{M \perp}\right|^{2}=F_{M}^{2} \sin ^{2} \alpha
\end{gathered}
$$

L'expression du rapport de flipping est alors :

$$
R=\frac{I^{+}}{I^{-}}=\frac{F_{N}^{2}+2 \sin ^{2} \alpha F_{N} F_{M}+\sin ^{2} \alpha F_{M}^{2}}{F_{N}^{2}-2 \sin ^{2} \alpha F_{N} F_{M}+\sin ^{2} \alpha F_{M}^{2}} .
$$

Considérant que l'angle $\alpha$ est un paramètre géométrique, bien mesuré au cours de l'expérience, il y a là une équation à une inconnue : la longueur du facteur de structure magnétique $\mathrm{F}_{\mathrm{M}}$. Cette quantité s'obtient donc par une équation du deuxième degré avec deux solutions correspondant une à $F_{M}>F_{N}$ et l'autre à $\mathrm{F}_{\mathrm{M}}<\mathrm{F}_{\mathrm{N}}$, le choix entre ces deux solutions étant en général évident.

Un autre cas intéressant se rencontre lorsque le structure est acentrique, mais avec l'aimantation toujours parfaitement alignée selon le champ. Les équations (6) et (7) sont toujours 
valables, mais avec des quantités complexes, et l'expression du rapport de flipping devient :

$$
\mathrm{R}=\frac{\mathrm{F}_{\mathrm{N}}^{\prime 2}+\mathrm{F}_{\mathrm{N}}^{\prime \prime 2}+2 \sin ^{2} \alpha\left(\mathrm{F}_{\mathrm{N}}^{\prime} \mathrm{F}_{\mathrm{M}}^{\prime}+\mathrm{F}_{\mathrm{N}}^{\prime \prime} \mathrm{F}_{\mathrm{M}}^{\prime \prime}\right)+\sin ^{2} \alpha\left(\mathrm{F}_{\mathrm{M}}^{\prime}{ }^{2}+\mathrm{F}_{\mathrm{M}}^{\prime}{ }^{2}\right)}{\mathrm{F}_{\mathrm{N}}^{\prime 2}+\mathrm{F}_{\mathrm{N}}^{\prime \prime}-\sin ^{2} \alpha\left(\mathrm{F}_{\mathrm{N}}^{\prime} \mathrm{F}_{\mathrm{M}}^{\prime}+\mathrm{F}_{\mathrm{N}}^{\prime \prime} \mathrm{F}_{\mathrm{M}}^{\prime \prime}\right)+\sin ^{2} \alpha\left(\mathrm{F}_{\mathrm{M}}^{\prime}{ }^{2}+\mathrm{F}_{\mathrm{M}}^{\prime \prime}\right)}
$$

c'est-à-dire une équation à deux inconnues $\mathrm{F}^{\prime} \mathrm{M}$ and $\mathrm{F}^{\prime \prime} \mathrm{M}$. Il y a dans ces rapports de flipping des informations très sensibles sur les facteurs de structure magnétiques, mais il n'est pas possible d'extraire, réflexion par réflexion, ces facteurs de structure à partir de cette équation (9). Comme il sera expliqué plus loin, il existe des traitements qui permettent de reconstituer les distributions de spin ou d'aimantation, directement à partir de ces facteurs de flipping expérimentaux.

Mais il faut rester prudent dans le cas de matériaux magnétiques très anisotropes, lorsque le champ magnétique appliqué est insuffisant pour aligner l'aimantation dans la direction verticale. Dans ce cas, les formules (6) et (7) ne sont pas valables et l'application des équations (8) ou (9) pour les cas centrique et acentrique entraînerait des résultats erronés. Ces cas se rencontrent lorsque des terres rares ou des actinides sont présents dans l'échantillon : leur anisotropie magnétique est souvent très grande et les champs magnétiques habituels peuvent être insuffisants pour aligner les moments magnétiques dans une direction qui n'est pas la direction facile. Ce cas se rencontre aussi pour des métaux de transition dont le moment orbital n'est pas bloqué.

Finalement, excepté dans ce dernier cas, la diffraction de neutrons polarisés mesure les rapports de flipping des réflexions de Bragg. Pour les structures centro-symétriques, les facteurs de structure magnétique se déduisent des rapports de flipping. Il reste maintenant à retrouver, à partir de là, la densité d'aimantation (ou la densité de spin).

\section{LA MÉTHODE DE FOURIER : UNE RECONSTRUCTION SANS MODÈLE}

\subsection{L'inversion de Fourier}

Comme le montre l'équation (1), les facteurs de structure magnétiques $F_{M}$ sont les coefficients de Fourier de la fonction périodique $\mathrm{M}(\mathbf{r})$. Il est alors tout à fait naturel d'appliquer l'inversion de Fourier pour obtenir la densité d'aimantation à partir de ces coefficients, c'est à dire des facteurs de structures magnétiques obtenus expérimentalement.

$$
\mathrm{M}(\mathrm{r})=\frac{1}{\mathrm{~V}} \sum_{Q} \mathrm{~F}_{\mathrm{M}}(\mathbf{Q}) \mathrm{e}^{-\mathrm{i} \mathbf{Q r}}
$$

où $\mathrm{V}$ est le volume de la maille élémentaire et où la somme sur $\square$ implique tous les vecteurs du réseau réciproque, c'est à dire une triple infinité pour les densités à 3 dimensions :

$$
\mathrm{M}(\mathrm{x}, \mathrm{y}, \mathrm{z})=\frac{1}{\mathrm{~V}} \sum_{\mathrm{h}} \sum_{\mathrm{k}} \sum_{\mathrm{l}} \mathrm{F}_{\mathrm{M}}(\mathrm{h}, \mathrm{k}, \mathrm{l}) \mathrm{e}^{-2 \pi \mathrm{i}(\mathrm{hx}+\mathrm{ky}+\mathrm{lz})}
$$

et une double infinité pour les projections de la densité selon une direction particulière, comme par exemple, le long de la direction $\mathbf{c}$ :

$$
\mathrm{M}(\mathrm{x}, \mathrm{y})=\frac{1}{\mathrm{~S}} \sum_{\mathrm{h}} \sum_{\mathrm{k}} \mathrm{F}_{\mathrm{M}}(\mathrm{h}, \mathrm{k}, 0) \mathrm{e}^{-2 \pi \mathrm{i}(\mathrm{hx}+\mathrm{ky})} .
$$

En pratique, il n'est pas possible de mesurer toutes les réflexions et les sommes (11) et (12) sont limitées à un nombre fini de facteurs de structure. Ce nombre est le plus souvent suffisant pour voir les détails recherchés dans la distribution. Une transformation inverse de Fourier pour une densité d'aimantation a été appliquée pour la première fois par Shull et Yamada ${ }^{(1)}$ dans le cas du fer. La figure 3 représente la section de cette densité par le plan (110) du cristal. 


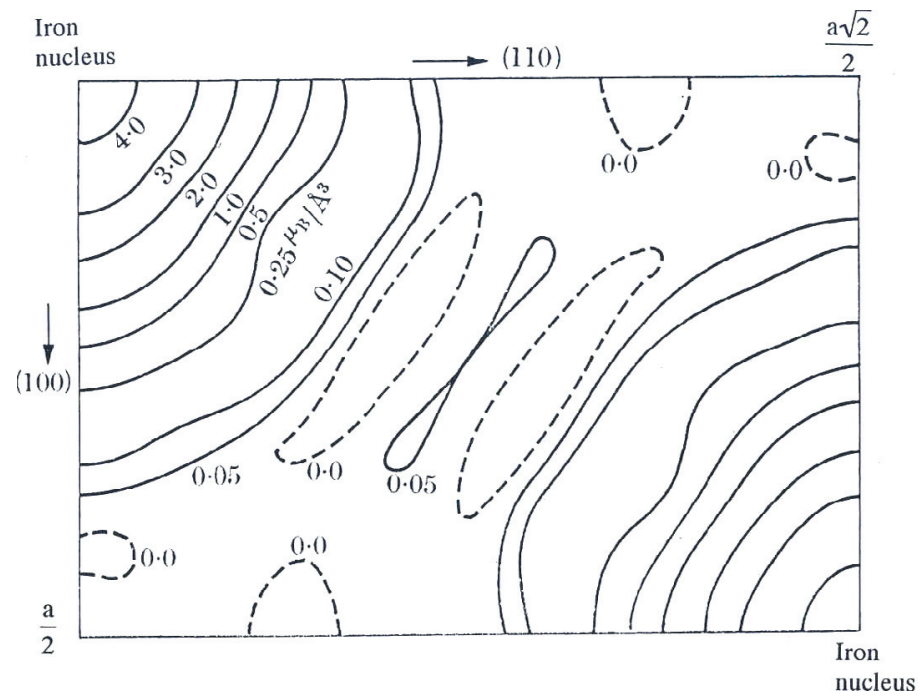

Figure 3. Densité d'aimantation dans le plan (110) d'un cristal de Fe, carte obtenue par inversion de Fourier ${ }^{(1)}$.

Cette methode a été utilisée systématiquement depuis pour pratiquement toutes les études avec des neutrons polarisés. Un exemple plus récent est représenté sur la figure 4 qui montre la coexistence d'atomes de cerium magnétiques et non magnétiques dans le composé $\mathrm{Ce}_{3} \mathrm{Sn}_{7}$, surstructure du système à valence intermédiaire $\mathrm{CeSn}_{3}$ (Boucherle et al. $\left.^{(2)}\right)$.

\subsection{Critiques de l'inversion de Fourier}

L'inversion de Fourier, si simple en pratique, est entachée d'un certain nombre de tares comme la limitation aux cristaux centro-symétriques, le caractère limité des données ou la non prise en considération des incertitudes expérimentales.

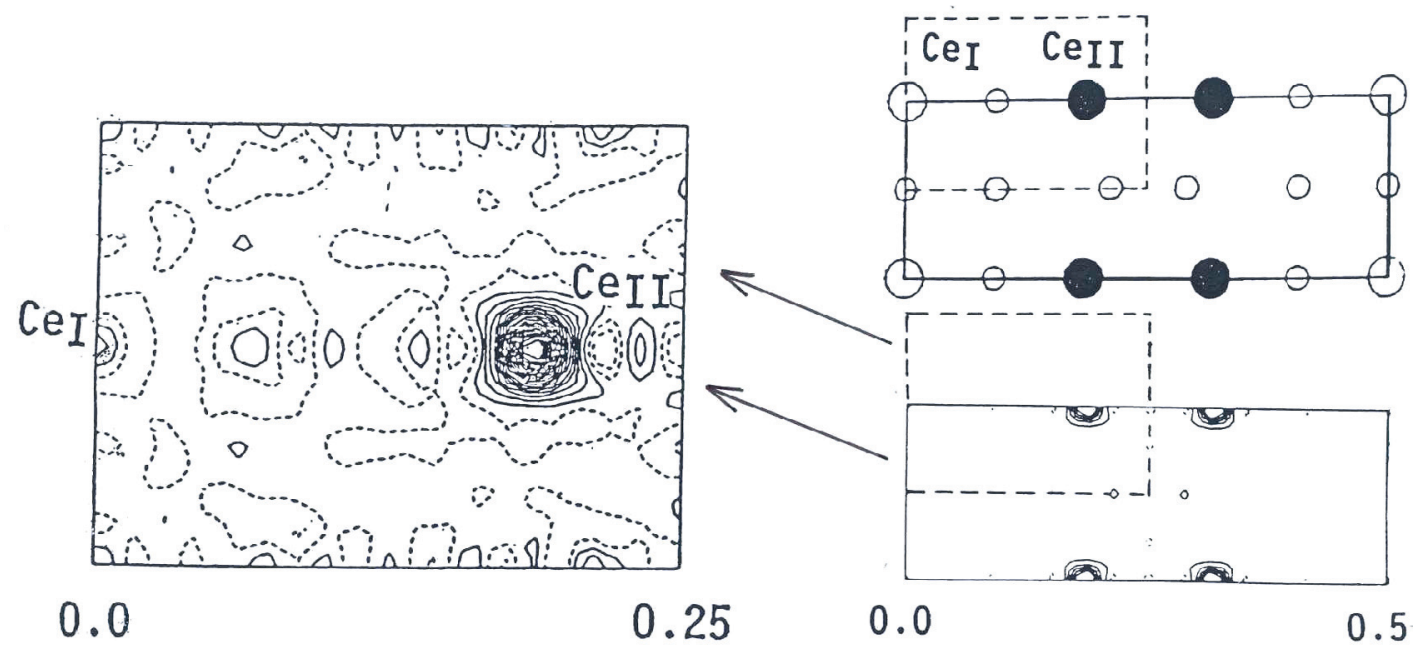

Figure 4. Densité d'aimantation projetée selon l'axe a du composé $\mathrm{Ce}_{3} \mathrm{Sn}_{7}$, densité obtenue par inversion de Fourier. L'atome $\mathrm{Ce}_{\mathrm{I}}$ n'est pas magnétique (comme dans le composé $\mathrm{CeSn}_{3}$ ) tandis que l'atome $\mathrm{Ce}_{\mathrm{II}}$ est magnétique $^{(2)}$. 


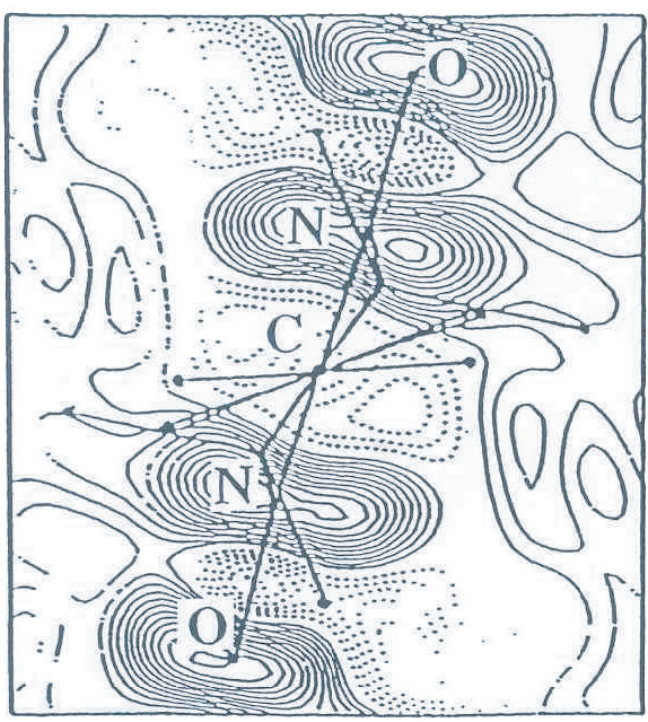

(a)

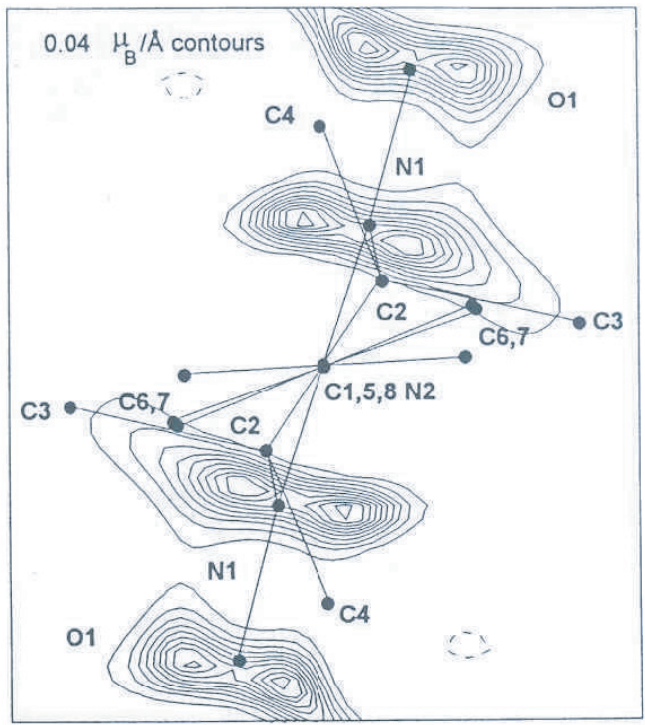

(b)

Figure 5. Densités de spin projetées selon l'axe $\mathbf{c}$ du $\beta$-pNPNN, obtenues à partir des 41 réflexions de type $(\mathrm{h}, \mathrm{k}, 0)$ (a) par inversion de Fourier, (b) par maximum d'entropie ${ }^{(3)}$.

\subsubsection{Limitation aux cristaux centro-symétriques}

Les formules (10), (11) et (12) qui permettent de construire la densité d'aimantation à partir des facteurs de structure magnétiques $F_{M}$ sont exactes, que les facteurs de structure soient réels ou complexes. Cependant, pour les structures non centriques où les $\mathrm{F}_{\mathrm{M}}$ sont complexes, nous avons vu qu'il n'était pas possible d'extraire directement les facteurs de structure magnétiques à partir des rapports de flipping $\mathrm{R}$. Il n'est alors pas possible d'obtenir une densité d'aimantation par inversion de Fourier.

Cette situation est très bien illustrée dans le cas de la phase $\beta$ du radical para-nitrophenyl nitronyl nitroxide ( $\beta$ p-NPNN), le premier exemple d'un composé ferromagnétique purement organique (température de Curie $\mathrm{T}_{\mathrm{C}}=0.65 \mathrm{~K}$ ). Il cristallise dans le groupe d'espace orthorhombique Fdd 2 qui est acentrique. Cependant, la projection de cette structure parallèlement à l'axe c, c'est à dire sur le plan $(\mathbf{a}, \mathbf{b})$ est centro-symétrique et les facteurs de structure $\mathrm{F}(\mathrm{h}, \mathrm{k}, 0)$ sont réels alors que les autres sont complexes. Une expérience de diffraction avec des neutrons polarisés ${ }^{(3)}$ a permis de mesurer les rapports de flipping de 246 réflexions, parmi lesquelles 41 étaient de type (h, k, 0). Il a alors été possible de construire par inversion de Fourier la projection de la densité de spin sur le plan (a, b) en utilisant l'expression (12) avec ces 41 réflexions. Le résultat est représenté sur la figure 5a qui montre que cette densité est surtout localisée sur le groupe ONCNO du radical, avec des orbitales de type p sur ces atomes, et une densité probablement négative (opposée au champ magnétique appliqué) sur le C central. Mais, à ce stade du traitement, il a été impossible de tirer avantage des informations contenues dans les 205 autres rapports de flipping mesurés pour obtenir une densité de spin à 3 dimensions par inversion de Fourier. D’autres méthodes ont dû être appliquées, qui seront développées plus loin.

\subsubsection{Données incomplètes}

L'inversion de Fourier, telle qu'elle est exprimée à travers les relations (10), (11) et (12) n'est exacte que si la somme sur $\square_{\mathrm{Q}}$ implique tous les points du réseau réciproque. Or le nombre de données mesurées 


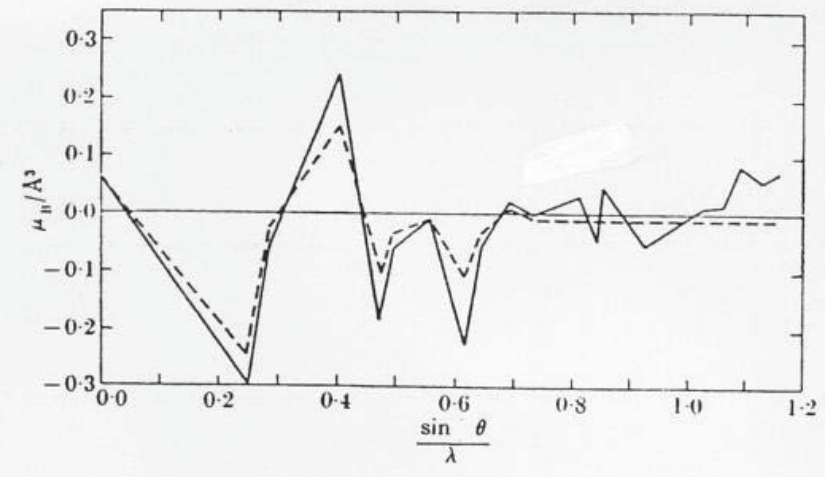

Figure 6. Convergence de la série de Fourier pour la densité d'aimantation au point ( $1 / 200)$ d'un cristal de nickel. Ligne continue : densité ponctuelle ; ligne brisée : densité moyenné $\mathrm{e}^{(6)}$.

est limité : d'une part il n'y a pas de mesures au delà d'une valeur limite $Q=Q_{\max }$, et d'autre part, à l'intérieur de cette sphère, certaines réflexions ne peuvent pas être mesurées parceque leurs amplitudes nucléaires sont trop faibles pour permettre une mesure valable du rapport de flipping. Pour ces raisons, les sommes (10), (11) et (12) sont en fait remplacées par des sommes partielles où les termes manquants ne sont pas négligeables a priori. Ceci entraîne à la fois des valeurs biaisées pour les densités calculées et des erreurs de troncature.

Pour réduire les biais dus à la limitation $\mathrm{Q}<\mathrm{Q}_{\max }$, il faut bien réaliser que de telles mesures ne permettent pas de reconstruire une distribution avec des détails de dimension $\mathrm{d}$ inférieure à $\mathrm{d}_{\min }{ }^{(4)}$ $\left(\mathrm{d}_{\min }=0.3 \lambda / \sin \theta_{\max }\right)$. Alors, au lieu de reconstruire une distribution ponctuelle $\mathrm{M}(\mathrm{x}, \mathrm{y}, \mathrm{z})$, densité au point $(\mathrm{x}, \mathrm{y}, \mathrm{z})$, on peut considérer la densité moyenne $\tilde{\mathrm{M}}(\mathrm{xyz})^{(5)}$, moyenne de la densité $\mathrm{M}(\mathrm{xyz})$ dans une boîte de volume $(2 \delta)^{3} \frac{\mathrm{V}}{\mathrm{abc}}$ autour du point (xyz); V, a, b et c correspondent au volume et aux paramètres de la maille élémentaire du cristal. Dans le cas cubique, cette densité moyenne s'exprime par :

$$
\tilde{\mathrm{M}}(\mathrm{x}, \mathrm{y}, \mathrm{z})=\int_{\mathrm{x}-\delta}^{x+\delta} \int_{y-\delta}^{y+\delta} \int_{z-\delta}^{x+\delta} \mathrm{M}(\mathrm{x}, \mathrm{y}, \mathrm{z}) \mathrm{dxdydz}
$$

$\tilde{\mathrm{M}}(\mathrm{x}, \mathrm{y}, \mathrm{z})$ s'obtient par une expression similaire à (10) où $\mathrm{F}_{\mathrm{M}}(\mathbf{Q})$ est remplacé par $\tilde{\mathrm{F}}_{\mathrm{M}}(\mathbf{Q})$ :

$$
\tilde{\mathrm{F}}_{\mathrm{M}}(\mathrm{h}, \mathrm{k}, \mathrm{l})=\left(\frac{\sin 2 \pi \mathrm{h} \delta}{2 \pi \mathrm{h} \delta}\right)\left(\frac{\sin 2 \pi \mathrm{k} \delta}{2 \pi \mathrm{k} \delta}\right)\left(\frac{\sin 2 \pi \mathrm{l} \delta}{2 \pi \mathrm{l} \delta}\right) \mathrm{F}_{\mathrm{M}}(\mathrm{h}, \mathrm{k}, \mathrm{l})
$$

La convergence de la série est plus rapide quand $\tilde{\mathrm{F}}_{\mathrm{M}}(\mathbf{Q})$ remplace $\mathrm{F}_{\mathrm{M}}(\mathbf{Q})$, et, plus grand est $\delta$, plus vite la série converge.

Un exemple de cette convergence accélérée est donné dans la figure 6 pour la densité d'aimantation au point $(1 / 2,0,0)$ de la maille cubique d'un cristal de nickel ${ }^{(6)}$. Les deux courbes, densité ponctuelle et densité moyennée, sont représentées en fonction de la valeur de coupure $(\sin \theta / \lambda)_{\max }$.

Des moyennes sur d'autres volumes adaptés à des problèmes spécifiques peuvent être utilisées. Elles entraînent la multiplication par d'autres fonctions d'apodisation pour transformer $F_{M}(\mathbf{Q})$ en $\tilde{F}_{M}(\mathbf{Q})$.

\subsubsection{Incertitudes expérimentales}

Les facteurs de structure expérimentaux $\mathrm{F}_{\mathrm{Mobs}}(\mathbf{Q})$ sont mesurés avec une certaine incertitude. Dans le traitement représenté par l'équation (10), les données sont introduites dans la sommation 
indépendamment de leur incertitude: un facteur de structure précis est traité exactement de la même façon qu'un facteur de structure imprécis. Cette part de l'information a été oubliée.

\subsubsection{Probabilités}

On peut considérer une carte reconstruite comme un modèle dans l'espace réel, modèle donné pour expliquer un ensemble de données $\mathrm{F}_{\mathrm{M} \text { obs }}(\mathbf{Q})$. Il est habituel, pour évaluer la probabilité d'un tel modèle, de comparer l'accord entre facteurs de structure observés et facteurs de structure calculés pour ce modèle. Pour ce faire, on définit une quantité $\chi^{2}$ telle que :

$$
\chi^{2}=\frac{1}{\mathrm{n}} \square \frac{1}{\sigma^{2}}\left|\mathrm{~F}_{\mathrm{Mcal}}(\mathbf{Q})-\mathrm{F}_{\mathrm{Mobs}}(\mathbf{Q})\right|^{2}
$$

où $\mathrm{n}$ est le nombre $\mathrm{d}$ 'observations indépendantes.

Pour le modèle obtenu par inversion de Fourier, $\mathrm{F}_{\mathrm{Mcal}}(\mathbf{Q})=\mathrm{F}_{\mathrm{Mobs}}(\mathbf{Q})$, quelles que soient les incertitudes expérimentales. Ceci montre que, parmi toutes les cartes possibles compatibles avec les données $\left(\chi^{2} \sim 1\right.$ or $\left.\chi^{2}<1\right)$, l'inversion de Fourier choisit celle qui donne exactement $\chi^{2}=0$ pour les réflexions mesurées, et exactement $\mathrm{F}_{\mathrm{Mcal}}=0$ pour celles qui n'ont pas été mesurées. C'est là la tare principale de la méthode.

\section{LA MÉTHODE DU MAXIMUM D'ENTROPIE : UNE RECONSTRUCTION SANS MODÈLE, MAIS INTELLIGENTE}

\subsection{Une approche bayesienne : les probabilités conditionnelles}

La reconstruction de cartes de densité de spin à partir d'un ensemble de données (les facteurs de structure magnétiques) peut être envisagée en termes de probabilités et de probabilités conditionnelles. Considérant toutes les cartes possibles, on va évaluer, pour chaque carte, sa probabilité, connaissant le jeu de facteurs de structure qui a été mesuré. On peut écrire une telle probabilité comme une probabilité conditionnelle $\mathrm{p}$ (map|data).

Il est alors utile d'introduire l'égalité de Bayes qui est très générale :

$$
\mathrm{p}(\mathrm{A} \mid \mathrm{B}) \mathrm{p}(\mathrm{B})=\mathrm{p}(\mathrm{B} \mid \mathrm{A}) \mathrm{p}(\mathrm{A})
$$

qui donne la probabilité a posteriori

$$
\mathrm{p}(\operatorname{map} \mid \text { data })=\frac{\mathrm{p}(\text { data } \mid \text { map }) \mathrm{p}(\text { map })}{\mathrm{p}(\text { data })} .
$$

Dans cette relation :

- p (data|map), la vraisemblance (likelihood), represente la probabilité du jeu de données expérimentales si la carte prise pour modèle correspond à la réalité. En d'autres termes, elle correspond à l' accord qui existe entre les $\mathrm{F}_{\mathrm{M} \text { obs }}$ et les $\mathrm{F}_{\mathrm{M} \text { cal }}$, et est exprimée par le $\chi^{2}$

- $\mathrm{p}$ (map), la probabilité a priori ou probabilité intrinsèque (prior probability), représente la probabilité de la carte, sans aucune référence avec le fait d'avoir mesuré des données expérimentales

- p (data) représente la probabilité des données expérimentales. Cette probabilité est égale à 1 lorsque que le jeu de données a été mesuré.

On a alors la relation:

$$
\mathrm{p}(\text { map } \mid \text { data })=\mathrm{p}(\text { data } \mid \text { map }) \mathrm{p}(\text { map })
$$

qui signifie que la probabilité d'une carte modèle, connaissant le jeu de données expérimentales, n'est pas seulement représenté par l'accord entre facteurs de structure calculés et observés, mais aussi par la probabilité intrinsèque de la carte. Il est clair que l'inversion de Fourier ignore complètement ce point. 
On peut dire que parmi toutes les reconstructions possibles qui sont compatibles avec les données, le maximum d'entropie choisit celle qui correspond à la plus grande priorité intrinsèque de la distribution.

\subsection{L'entropie d'une distribution}

La probabilité intrinsèque d'une carte, $\mathrm{p}(\mathrm{map})$, peut s'exprimer en terme d'entropie. Ce concept a été introduit par Boltzmann pour exprimer la probabilité d'une configuration dans l'espace des phases (un espace à $6 \mathrm{~N}$ dimensions représentant les positions et les vitesses) de $\mathrm{N}$ particules. Il a montré que toutes les configurations n'avaient pas les mêmes chances d'exister : la configuration la plus probable étant celle qui rendait maximum la quantité appelée entropie :

$$
\mathrm{S}_{\mathrm{B}}=-\mathrm{N} \underset{i}{\square} \mathrm{P}_{\mathrm{i}} \log \mathrm{p}_{\mathrm{i}}
$$

où $\mathrm{p}_{\mathrm{i}}=\mathrm{Ni} / \mathrm{N}$ et où $\mathrm{Ni}$ était l'occupation de la cellule $\mathrm{i}$. Cette approche, qui n'utilise pas de loi dynamique autre que la conservation d'énergie, apparaît comme particulièrement efficace dans la mesure où elle introduit les concepts de température absolue, de distribution de Boltzmann et beaucoup d'autres conséquences.

Le concept d'entropie a été ensuite généralisé par Shannon ${ }^{(7)}$ à la théorie de l'information. Le problème des kangourous ${ }^{(8)}$ illustre bien la signification de l'entropie dans ce contexte : "On sait que $50 \%$ des kangourous habitant dans une île déserte ont les yeux bleus (YB) et que 40\% d'entre eux sont gauchers $(\mathrm{G})$. La question à laquelle il faut répondre est, en l'absence de toute autre information, quelle est la proportion de ceux qui à la fois ont des yeux bleus et sont gauchers". La solution n'est pas unique et toutes les réponses entre $0 \%$ et $40 \%$ sont possibles; mais ces réponses n' ont pas la même probabilité. Restreignons-nous, pour être plus simple, à 10 kangourous dont les noms seraient $\mathrm{A}, \mathrm{B}$, C, ... I, J. La Figure 7 représente 3 répartitions possibles, respectant toutes $50 \%$ (YB) et $40 \%$ (G), mais les répartitions (a) et (b) correspondent à une connfiguration $\left(\begin{array}{ll}1 & 4 \\ 3 & 2\end{array}\right)$ tandis que (c) correspond à la configuration $\left(\begin{array}{ll}2 & 3 \\ 2 & 3\end{array}\right)$. Le nombre de répartitions différentes de kangourous qui donnent la même configuration (nombre de complexions) se calcule facilement en analyse combinatoire. Il est reporté dans le tableau 1. Il est clair que, en l'absence d'autres informations, la configuration $\left(\begin{array}{ll}2 & 3 \\ 2 & 3\end{array}\right)$ est plus probable que les autres. Remarquons que cette réponse avec $20 \%$ de kangourous à la fois (YB) et $(\mathrm{G})$ correspond au découplage entre les deux informations partielles, ce qui est le plus probable en l'absence d'autres informations.

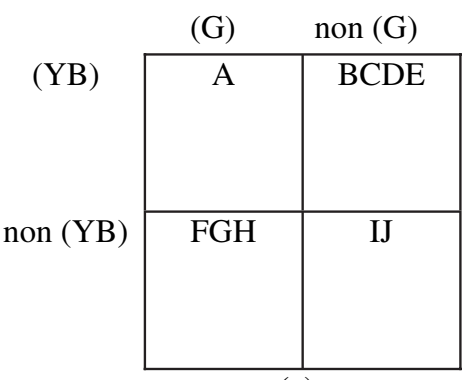

(a)
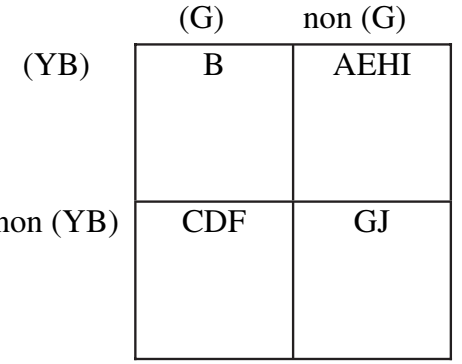

(b)
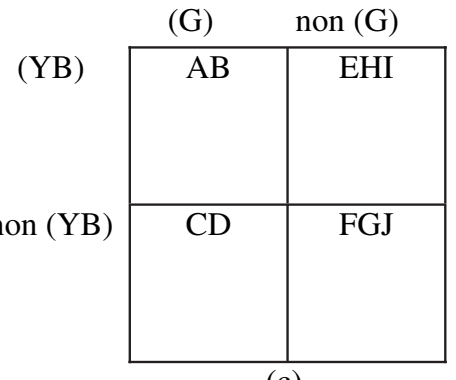

(c)

Figure 7. Trois repartitions possibles des 10 kangourous. Les répartitions (a) et (b) correspondent à une configuration $\left(\begin{array}{ll}1 & 4 \\ 3 & 2\end{array}\right)$ tandis que (c) corresponds à $\left(\begin{array}{ll}2 & 3 \\ 2 & 3\end{array}\right)$. 
Tableau 1. Nombre de complexions pour les differentes configurations des 10 kangourous.

\begin{tabular}{|c|c|}
\hline Configuration & Nombre de complexions \\
\hline$\left(\begin{array}{ll}0 & 5 \\
4 & 1\end{array}\right)$ & $\frac{10 !}{5 ! 4 ! 1 !}=1260$ \\
\hline$\left(\begin{array}{ll}1 & 4 \\
3 & 2\end{array}\right)$ & $\frac{10 !}{4 ! 3 ! 2 ! 1 !}=12600$ \\
\hline$\left(\begin{array}{ll}2 & 3 \\
2 & 3\end{array}\right)$ & $\frac{10 !}{3 ! 3 ! 2 ! 2 !}=25200$ \\
\hline$\left(\begin{array}{ll}3 & 2 \\
1 & 4\end{array}\right)$ & $\frac{10 !}{4 ! 3 ! 2 ! 1 !}=12600$ \\
\hline$\left(\begin{array}{ll}4 & 1 \\
0 & 5\end{array}\right)$ & $\frac{10 !}{5 ! 4 ! 1 !}=1260$ \\
\hline
\end{tabular}

Pour généraliser à des répartitions sur $\mathrm{M}$ cellules, le nombre de complexions d'une configuration donnée est :

$$
\mathrm{W}=\frac{\mathrm{N} !}{\left(\mathrm{N}_{1}\right) !\left(\mathrm{N}_{2}\right) ! \ldots\left(\mathrm{N}_{\mathrm{M}}\right) !}
$$

qui devient, après application de la formule de Stirling pour les grands nombres :

$$
\mathrm{W}=\frac{\mathrm{N}^{\mathrm{N}}}{\left(\mathrm{N}_{1}\right)^{\mathrm{N}_{1}}\left(\mathrm{~N}_{2}\right)^{\mathrm{N}_{2}} \ldots\left(\mathrm{N}_{\mathrm{M}}\right)^{\mathrm{N}_{\mathrm{M}}}}=\frac{1}{\left(\mathrm{p}_{1}\right)^{\mathrm{N}_{1}}\left(\mathrm{p}_{2}\right)^{\mathrm{N}_{2}} \ldots\left(\mathrm{p}_{\mathrm{M}}\right)^{\mathrm{N}_{\mathrm{M}}}}
$$

avec $\mathrm{p}_{\mathrm{i}}=\mathrm{Ni} / \mathrm{N}$.

La repartition qui maximise $\mathrm{W}$ maximise également son logarithme, c'est à dire l'entropie de la distribution :

$$
\log \mathrm{W}=\mathrm{S}_{\mathrm{B}}=\mathrm{NS}
$$

où

$$
S=-\sum_{i} p_{i} \log p_{i}
$$

\subsection{Application à la densité de spin d'une distribution ${ }^{(9,10)}$}

Pour pouvoir définir l'entropie d'une densité de spin (ou d'aimantation), cette fonction continue est rendue discrète en divisant la maille cristallographique en sous-unités i dans lesquelles la densité est supposée constante : $\mathrm{M}_{\mathrm{i}}=\mathrm{M}\left(\mathbf{r}_{\mathrm{i}}\right)$. Comme la densité de spin peut aussi bien être négative que positive, il faut considérer une double distribution de quantités positives : $\mathrm{M}_{\mathrm{i}}=\mathrm{M}^{+}\left(\mathbf{r}_{\mathrm{i}}\right)$ et $\mathrm{M}_{\mathrm{i}+\mathrm{N}}=\mathrm{M}^{-}\left(\mathbf{r}_{\mathrm{i}}\right)$, avec $\mathrm{M}\left(\mathbf{r}_{\mathrm{i}}\right)=\mathrm{M}^{+}\left(\mathbf{r}_{\mathrm{i}}\right)-\mathrm{M}^{-}\left(\mathbf{r}_{\mathrm{i}}\right)$. On définit ensuite des densités normalisées :

$$
\mathrm{p}_{\mathrm{i}}=\frac{\mathrm{M}_{\mathrm{i}}}{\sum_{\mathrm{j}=1}^{2 \mathrm{~N}} \mathrm{M}_{\mathrm{j}}}
$$

L'entropie de chaque distribution est alors définie par l'équation (22).

La distribution de spin la plus probable est celle qui a le maximum d'entropie parmi les distributions qui sont compatibles avec les données expérimentales. Pour l'obtenir, on part d'une distribution plate, on calcule les facteurs de structure $\mathrm{F}_{\mathrm{M} \text { cal }}$ qui lui correspondent, et on évalue l'accord avec les données par la valeur du $\chi^{2}$ défini par l'équation (5). La distribution de départ est ensuite affinée de façon à amener la valeur du $\chi^{2}$ au voisinage de l'unité, mais pas plus bas, et en même temps, d'atteindre la plus 
haute valeur possible pour l'entropie. Le programme d'affinement utilise la bibliothèque MEMSYS ${ }^{(11)}$. Ces programmes d'affinement sont opérationnels à l'ILL, au LLB et au CEA-Grenoble.

Un premier exemple de reconstruction entropique est la projection de la densité de spin obtenue à partir de 41 facteurs de structure réels du type (hk0) du $\beta$ p-NPNN ${ }^{(3)}$. Cette reconstruction est comparée à l'inversion de Fourier sur les figures $5 \mathrm{a}$ et $5 \mathrm{~b}$ : tous les détails fallacieux de la reconstruction de Fourier ont disparu, ainsi que la densité négative sur le carbone central. De plus, sur la nouvelle carte, la forme des orbitales des atomes $\mathrm{O}, \mathrm{N}, \mathrm{N}, \mathrm{O}$ est bien plus claire et caractéristique des orbitales $2 \mathrm{p}$. On peut aussi voir que ces orbitales $2 p$ ne sont pas exactement centrées sur les noyaux $\mathrm{N}$ et $\mathrm{O}$, mais poussées vers l'extérieur du segment NO, traduisant ainsi le caractère antiliant de la fonction d'onde moléculaire.

Un autre exemple est illustré sur la Figure 8. Il compare les projections de la densité de spin dans le supraconducteur à haut $\mathrm{T}_{\mathrm{C}} \mathrm{YBa}_{2} \mathrm{Cu}_{3} \mathrm{O}_{7}$ lorsque les données sont traitées par inversion de Fourier et par reconstruction entropique. La comparaison parle d'elle même : les artefacts de la première méthode qui sont essentiellement dus aux effets de troncature ont été supprimés dans la reconstruction entropique. Fondamentalement, toute densité qui y apparaît doit être considérée comme significative et résultant des données expérimentales. Ceci vient du fait que de telles reconstructions cherchent à être aussi lisses que possible, tout en étant compatibles avec les données, compte tenu de la barre d'erreur de ces dernières.

L'extension des reconstructions entropiques aux structures acentriques a représenté un développement important de la méthode ${ }^{(13,14)}$. Dans ce cas, la densité de spin (ou d'aimantation) est reconstruite, non pas à partir des facteurs de structure magnétiques, mais directement à partir des rapports de flipping, selon la relation (5). De tels programmes de reconstruction sont opérationnels au LLB et au CEA-Grenoble. Une illustration de ce développement est donné par la la projection de la

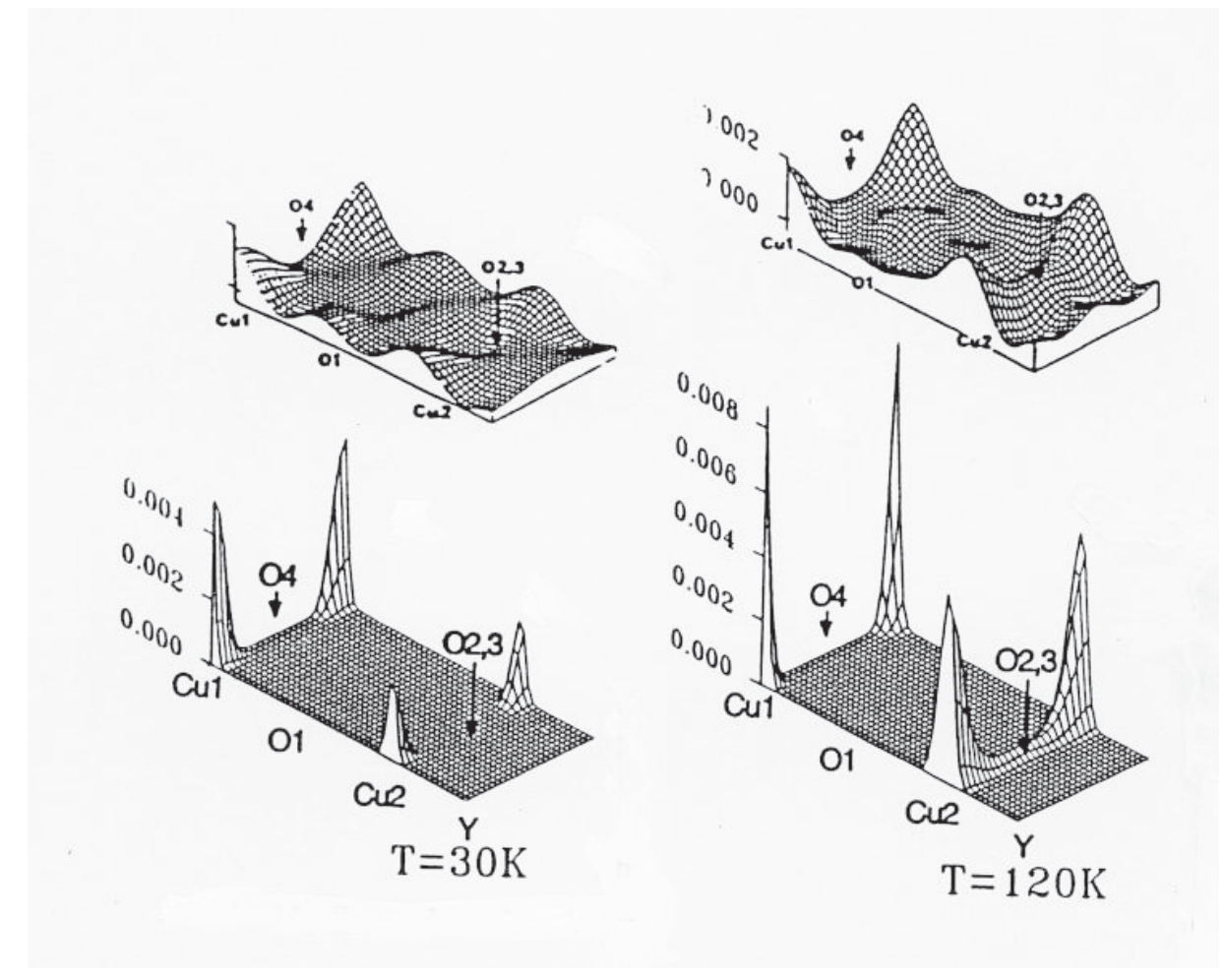

Figure 8. Densité de spin de $\mathrm{YBa}_{2} \mathrm{Cu}_{3} \mathrm{O}_{7}$ en dessus et en dessous de la temperature critique $\mathrm{T}_{\mathrm{C}}=90 \mathrm{~K}$. Les reconstructions du haut ont été obtenues par inversion de Fourier, celles du bas par maximum d'entropie ${ }^{(12)}$. 


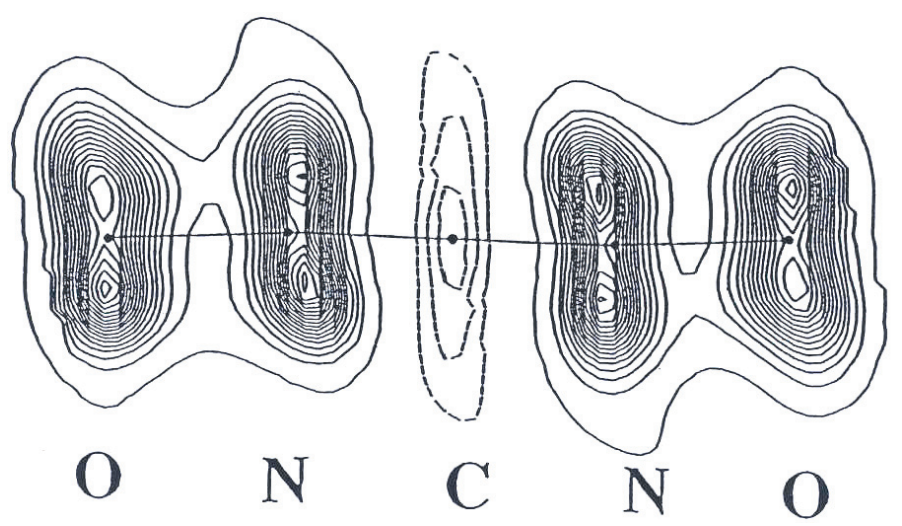

Figure 9. Densité de spin projetée le long de l'axe $\mathbf{c}$ du $\beta$-pNPNN, reconstruction entropique à partir des rapports de flipping des 246 reflexions mesurées dans cette structure acentrique ${ }^{(14)}$.

densité de spin sur le plan $\mathbf{a}, \mathbf{b}$ du $\beta$ p-NPNN, reconstruction à partir des 246 réflexions mesurées, les 41 (hk0) et les 205 autres. Cette reconstruction est représentée sur la figure 9. Maintenant, la densité négative sur le carbone central est indéniablement là et elle a la forme allongée caractéristique des orbitales $2 \mathrm{p}$.

\section{MODÉLISATION DE LA FONCTION D'ONDE MAGNÉTIQUE}

Une façon a priori simple et pratique de reconstruire la densité de spin, tout en évitant les problèmes liés à la transformée de Fourier inverse, est de modéliser cette densité de spin et de déterminer les paramètres de cette modélisation par un affinement à partir des données expérimentales. Une fois ces paramètres connus, la reconstruction de la densité de spin est immédiate.

Le premier modèle, le plus naturel, pour représenter une densité de spin, est de la considérer comme le carré de la fonction d'onde des électrons magnétiques. Les facteurs de structure s'écrivent alors :

$$
F_{M}=\left\langle\Psi\left|e^{i Q r}\right| \Psi\right\rangle=\int \Psi^{*} e^{i Q r} \Psi d^{3} r
$$

où $|\Psi\rangle$ est la fonction d'onde moléculaire qu'on écrit en général :

$$
|\Psi\rangle=\sum_{\text {atomes }} \alpha_{\mathrm{j}}\left|\varphi_{j}\right\rangle
$$

avec une somme qui porte sur tous les atomes magnétiques.

Il y a deux sortes de termes dans l'expression de $\mathrm{F}_{\mathrm{M}}$ :

- des intégrales à un centre

$$
\int \varphi_{\mathrm{j}}^{*}(\mathbf{r}) \mathbf{e}^{\mathrm{i} \mathbf{Q r}} \varphi_{\mathrm{j}}(\mathbf{r}) \mathbf{d}^{3} \mathbf{r}
$$

qui représentent la contribution principale

- des intégrales à deux centres

$$
\int \varphi_{\mathrm{j}^{\prime}}^{*}(\mathbf{r}) \mathbf{e}^{\mathrm{i} \mathbf{Q r}} \varphi_{\mathrm{j}}(\mathbf{r}) \mathbf{d}^{3} \mathbf{r}
$$

qui ne sont que des termes correcteurs correspondant aux recouvrements d'orbitales entre atomes voisins. 
Dans ce qui suit nous nous restreindrons aux intégrales à un centre. Nous exprimerons les amplitudes diffusées par les atomes porteurs d'une certaine densité d'aimantation en terme de facteur de forme magnétique. Nous considérerons successivement le cas des électrons p et d pour lesquels l'aimantation provient essentiellement du spin, et le cas des électrons f (terres rares et actinides) où le spin et l'orbite se couplent pour donner un moment angulaire total.

On peut trouver le traitement général du facteur de forme dans Marshall et Lovesey ${ }^{(15)}$, dans Lovesey $^{(16)}$ ou dans Balcar et Lovesey ${ }^{(17)}$.

\subsection{Facteur de forme des électrons p ou d pour une densité d'aimantation due au spin uniquement}

Dans le cas d'une densité d'aimantation due uniquement au spin, on peut écrire le facteur de forme magnétique $\mathbf{f}(\mathbf{Q})$ de la façon suivante :

$$
\mu \mathrm{f}(\mathrm{Q})=\int \varphi^{*} \mathrm{e}^{\mathrm{i} \mathbf{Q r}} \varphi \mathrm{d}^{3} \mathrm{r}
$$

où $\mu$ est le moment magnétique de l'atome. La fonction d'onde atomique $\varphi(\mathbf{r})$ se développe en une partie radiale $\mathrm{R}(\mathrm{r})$ et une partie angulaire :

$$
\varphi(\mathbf{r})=\sum_{\ell} \mathrm{R}_{\mathrm{a}}^{\ell}(\mathrm{r}) \sum_{\mathrm{m}=-\ell}^{\ell} \mathrm{a}_{\ell \mathrm{m}} \mathrm{Y}_{\ell}^{\mathrm{m}}(\theta, \varphi)
$$

où $\theta, \varphi$ sont les coordonnées angulaires de $\mathbf{r}$ et où les $\mathrm{Y}_{\ell}^{\mathrm{m}}$ sont les harmoniques sphériques habituelles.

La valeur de $\ell$ vaut $0,1,2 \ldots$ pour les électrons $s, p, d \ldots$ En général, une seul valeur de $\ell$ est concernée par le magnétisme.

On peut développer l'exponentielle de (27) par

$$
\mathrm{e}^{\mathrm{i} \mathbf{Q r}}=4 \pi \sum_{\mathrm{L}=0}^{\infty} \mathrm{i}^{\mathrm{L}} \mathrm{j}_{\mathrm{L}}(\mathrm{Qr}) \sum_{\mathrm{M}=-\mathrm{L}}^{\mathrm{L}} \mathrm{Y}_{\mathrm{L}}^{\mathrm{M}}+\mathrm{L}\left(\theta_{Q}, \varphi_{Q}\right)^{*} \mathrm{Y}_{\mathrm{L}}^{\mathrm{M}}(\theta, \varphi)
$$

où $\theta_{Q}, \varphi_{Q}$ sont les coordonnées angulaires de $\mathbf{Q}$ et où les $\mathrm{j}_{\mathrm{L}}(\mathrm{z})$ sont les fonctions de Bessel sphériques (voir l'appendice).

En introduisant ce développement dans (27) on obtient :

$$
\mathrm{f}(\mathbf{Q})=\sum_{\mathrm{L}=0}^{\infty}\left\langle\mathrm{j}_{\mathrm{L}}(\mathrm{Qr})\right\rangle \sum_{\mathrm{M}=-\mathrm{L}}^{\mathrm{L}} \mathrm{C}_{\mathrm{LM}} \mathrm{Y}_{\mathrm{L}}^{\mathrm{M}}\left(\theta_{Q}, \varphi_{Q}\right)^{*}
$$

où les $\left\langle\mathrm{j}_{\mathrm{L}}(\mathrm{Q})\right\rangle$ sont les integrales radiales des électrons magnétiques, c'est à dire une forme de transformée de Fourier, par fonction de Bessel interposées, des fonctions radiales de ces électrons.

$$
\left\langle\mathrm{j}_{\mathrm{L}}(\mathrm{Q})\right\rangle=\int_{0}^{\infty} \mathrm{r}^{2}\left(\mathrm{R}_{\mathrm{a}}^{\ell}(\mathrm{r})\right) \mathrm{j}_{\mathrm{L}}(\mathrm{Qr}) \mathrm{dr} .
$$

Les intégrales radiales ont été tabulées par J. Brown ${ }^{(18)}$ pour les électrons $3 \mathrm{~d}$ et $4 \mathrm{~d}$ des métaux de transition ainsi que pour les électrons $4 \mathrm{f}$ des terres rares et $5 \mathrm{f}$ des actinides.

Il est courant en magnétisme moléculaire d'exprimer la partie radiale de la fonction d'onde à l'aide des fonctions atomiques de Slater :

$$
\mathrm{R}_{\mathrm{a}}^{\ell}(\mathrm{r})=\frac{(2 \alpha)^{\left(\mathrm{n}_{\mathrm{a}}+\frac{1}{2}\right)}}{\sqrt{\left(2 \mathrm{n}_{\mathrm{a}}\right) !}} \mathrm{r}^{\left(\mathrm{n}_{\mathrm{a}}-1\right)} \mathrm{e}^{-\alpha \mathrm{r}}
$$

expression qui dépend du nombre quantique principal $\mathrm{n}_{a}$ et où l'exposant de Slater $\alpha$, characteristique des deux nombres quantiques $\mathrm{n}_{\mathrm{a}}$ et $\ell$ de l'orbitale atomique, ont été calculés et tabulés, par exemple 


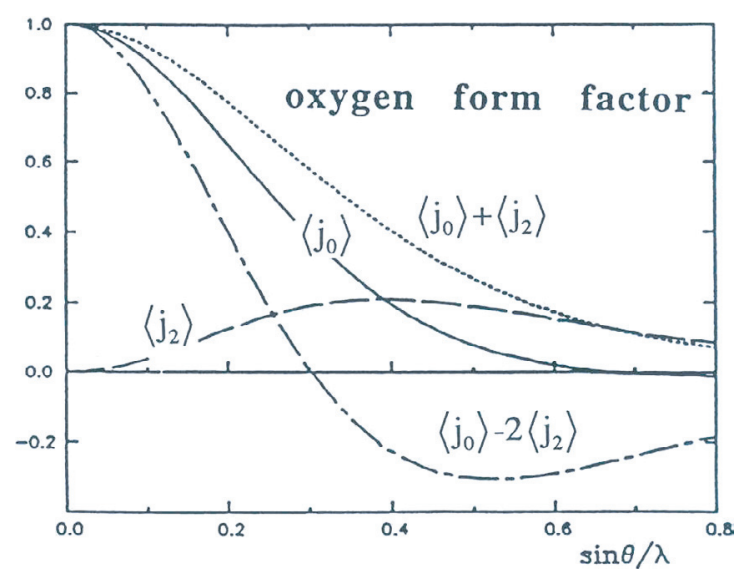

Figure 10. Intégrales radiales et limites du facteur de forme $\mathrm{f}(\mathrm{Q})$ calculés pour l'oxygène.

par Clementi et Roetti ${ }^{(19)}$. Dans ce formalisme, les intégrales radiales s'expriment par des fonctions analytiques de $\mathrm{Q}$ et de $\alpha$ (voir dans l'appendice les expressions de $\left\langle\mathrm{j}_{0}\right\rangle$ et de $\left\langle\mathrm{j}_{2}\right\rangle$ pour les électrons $2 \mathrm{p}$ ainsi que celles de $\left\langle\mathrm{j}_{0}\right\rangle,\left\langle\mathrm{j}_{2}\right\rangle$ et de $\left\langle\mathrm{j}_{4}\right\rangle$ pour les électrons $\left.3 \mathrm{~d}\right)$. La figure 9 montre ces intégrales pour les électrons $2 \mathrm{p}$ de l'oxygène.

Dans l'expression (30), les coefficients $\mathrm{C}_{\mathrm{LM}}$ sont donnés par :

$$
\mathrm{C}_{\mathrm{LM}}=\mathrm{i}^{\mathrm{L}}(2 \ell+1)[4 \pi(2 \mathrm{~L}+1)]^{\frac{1}{2}}\left(\begin{array}{lll}
\ell & \mathrm{L} & \ell \\
0 & 0 & 0
\end{array}\right) \sum_{\mathrm{mm}^{\prime}}(-1)^{\mathrm{m}_{\ell \mathrm{m}}} \mathrm{a}_{\ell \mathrm{m}^{\prime}}^{*}\left(\begin{array}{ccc}
\ell & \mathrm{L} & \ell \\
-\mathrm{m} & \mathrm{M} & \mathrm{m}^{\prime}
\end{array}\right)
$$

où les symboles $3 \mathrm{j}\left(\begin{array}{l}\mathrm{a} b \mathrm{c} \\ \mathrm{d} \text { e f }\end{array}\right)$ sont étroitement liés aux coefficients de Clebsch-Gordan.

À cause des relations triangulaires qui existent pour les symboles $3 \mathrm{j}$ :

$$
\left\{\begin{array}{c}
\mathrm{L} \leq 2 \ell \\
-\mathrm{m}+\mathrm{M}+\mathrm{m}^{\prime}=0
\end{array}\right\}
$$

l'expression du facteur de forme se limite à :

$$
\begin{array}{ll}
\text { pour des électrons p }(\ell=1) & \mathrm{f}(\mathbf{Q})=\left\langle\mathrm{j}_{0}(\mathrm{Q})\right\rangle+\mathrm{A}\left(\theta_{Q}, \varphi_{\mathrm{Q}}\right)\left\langle\mathrm{j}_{2}(\mathrm{Q})\right\rangle \\
\text { pour des électrons d }(\ell=2) & \mathrm{f}(\mathbf{Q})=\left\langle\mathrm{j}_{0}(\mathrm{Q})\right\rangle+\mathrm{A}\left(\theta_{Q}, \varphi_{\mathrm{Q}}\right)\left\langle\mathrm{j}_{2}(\mathrm{Q})\right\rangle+\mathrm{B}\left(\theta_{\mathrm{Q}}, \varphi_{\mathrm{Q}}\right)\left\langle\mathrm{j}_{4}(\mathrm{Q})\right\rangle .
\end{array}
$$

Connaissant la fonction d'onde des électrons magnétiques, le calcul du facteur de forme se déduit des formules (30) et (33). En particulier, pour une orbitale $2 p$ de l'oxygène alignée selon $\mathrm{Oz}$ (orbitale $2 \mathrm{p}_{\mathrm{z}}$ ), le facteur de forme s'écrit :

$$
\mathrm{f}(\mathbf{Q})=\left\langle\mathrm{j}_{0}(\mathrm{Q})\right\rangle+\left(1-3 \cos ^{2} \theta_{Q}\right)\left\langle\mathrm{j}_{2}(\mathrm{Q})\right\rangle .
$$

C'est un facteur de forme très anisotrope, dépendant fortement de $\theta_{Q}$, l'angle entre le vecteur de diffusion $\mathbf{Q}$ et la direction $\mathrm{Oz}$ de l'orbitale. Il s'inscrit entre les deux fonctions $\left\langle\mathrm{j}_{0}(\mathrm{Q})\right\rangle-2\left\langle\mathrm{j}_{2}(\mathrm{Q})\right\rangle$ et $\left\langle\mathrm{j}_{0}(\mathrm{Q})\right\rangle+\left\langle\mathrm{j}_{2}(\mathrm{Q})\right\rangle$ pour $\theta_{Q}=0$ et pour $\theta_{Q}=\pi / 2$, comme on peut le voir sur la figure 10 .

D'une façon générale, la modélisation de la densité de spin à l'aide d'une fonction d'onde électronique s'est avéré être une méthode simple et utile. On affine les paramètres ajustables comme les coefficients $\mathrm{a}_{\ell m}$ de la fonction d'onde, ou les coefficients de Slater $\alpha$ qui jouent sur l'extension de la partie radiale $\mathrm{R}(\mathrm{r})$. Ce modèle est tout à fait adapté pour détecter les faibles densités de spin qui existent sur certains atomes. 


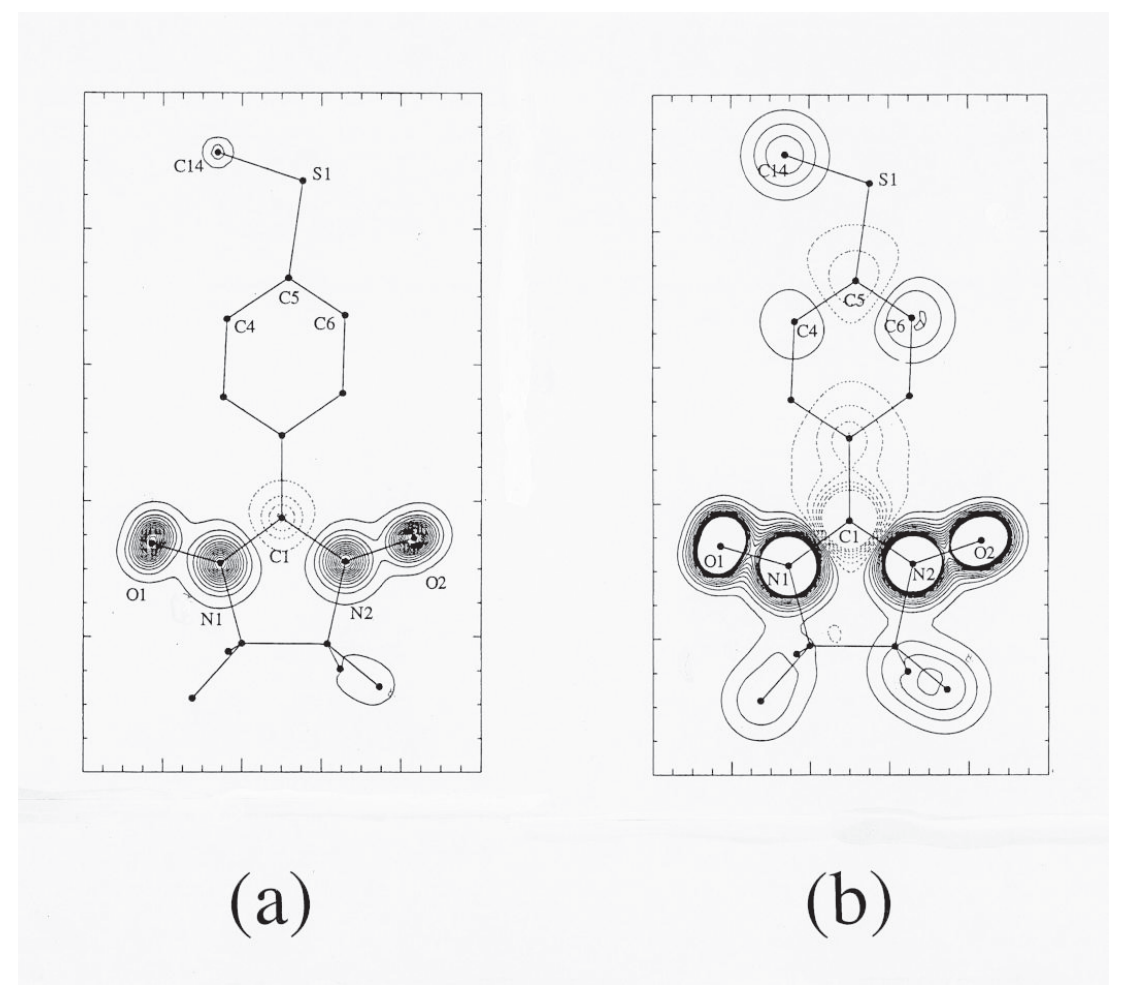

Figure 11. Radical Nit(SMe)Ph : projection sur le plan moyen du groupe ONCNO de la densité de spin ; densité modélisée à partir de la fonction d'onde : (a) hauts contours (pas $0.04 \mu_{\mathrm{B}} / \mathrm{A}^{2}$ ); (b) bas contours (pas $0.006 \mu_{\mathrm{B}} / \mathrm{A}^{2}$ ).

La figure 11 donne l'exemple d'un tel affinement. Il s'agit de la densité de spin du radical libre $\mathrm{Nit}(\mathrm{SMe}) \mathrm{Ph}$, dont les fonctions d'onde atomiques qui servent à sa modélisation ont été affinées à partir des facteurs de structure expérimentaux ${ }^{(20)}$.

La majeure partie de la densité de spin est portée par le fragment ONCNO, avec un signe négatif sur l'atome de carbone central. Une densité de spin avec alternance de signes, nettement plus faible que sur le fragment précédent, est visible sur le reste de la molécule. On peut voir sur la figure 12 que les interactions avec les molécules voisines ont incliné les orbitales p des atomes $\mathrm{O} 1$ et $\mathrm{O} 2$, qui ne sont plus perpendiculaires au plan ONCNO.

Des programmes d'affinement pour une modélisation de la densité de spin en fonctions d'onde atomiques existent à l'ILL et au LLB, autour des diffractomètres à neutrons polarisés. Ce sont des programmes restreints aux structures centro-symétriques car elles affinent les paramètres du modèle à partir des valeurs expérimentales du facteur de structure magnétique $\mathrm{F}_{\mathrm{M}}$. Il existe aussi d'autres programmes, adaptés aux structures acentriques pour lesquelles l'affinement se fait à partir des rapports de flipping R.

\subsection{Facteurs de forme des électrons p et $\mathrm{d}$ : la contribution du moment orbital}

Lorsque le moment cinétique orbital n'est pas complètement bloqué, il apporte sa contribution au moment magnétique qui comprend alors une partie orbitale en plus de sa partie spin.

$$
\begin{gathered}
\mu=\mu_{\mathrm{s}}+\mu_{\mathrm{L}} \\
\mathrm{f}(\mathbf{Q})=\mathrm{a}_{\mathrm{s}} \mathrm{f}_{\mathrm{s}}(\mathbf{Q})+\mathrm{a}_{\mathrm{L}} \mathrm{f}_{\mathrm{L}}(\mathbf{Q})
\end{gathered}
$$




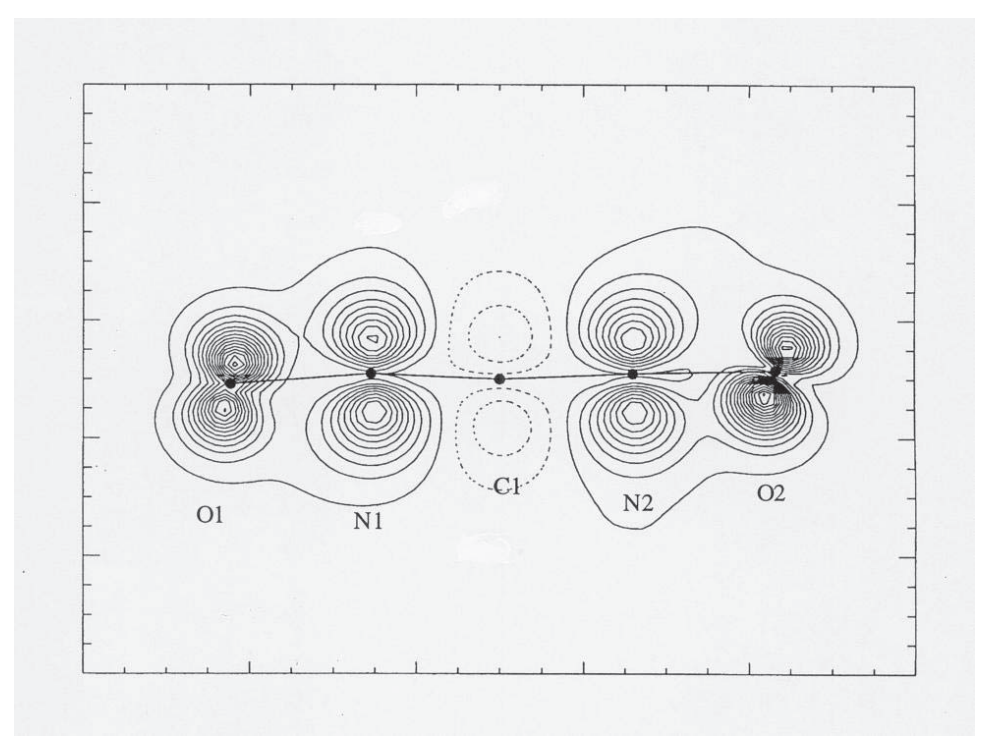

Figure 12. Radical $\mathrm{Nit}(\mathrm{SMe}) \mathrm{Ph}$ : projection sur un plan perpendiculaire au plan moyen du groupe ONCNO de la densité de spin ; densité modélisée à partir de la fonction d'onde (pas $0.03 \mu_{\mathrm{B}} / \mathrm{A}^{2}$ ).

où $\mathrm{a}_{\mathrm{S}}$ et $\mathrm{a}_{\mathrm{L}}$ sont les proportions de spin et d'orbite du moment magnétique. Dans une approximation sphérique les facteurs de forme de spin et d'orbite s'écrivent :

$$
\begin{gathered}
\mathrm{f}_{\mathrm{S}}(\mathbf{Q})=\left\langle\mathrm{j}_{0}\right\rangle \\
\mathrm{f}_{\mathrm{L}}(\mathbf{Q})=\left\langle\mathrm{j}_{0}\right\rangle+\left\langle\mathrm{j}_{2}\right\rangle
\end{gathered}
$$

ce qui donne pour le facteur de forme total :

$$
f(Q)=\left\langle j_{0}\right\rangle+a_{L}\left\langle j_{2}\right\rangle \text {. }
$$

Notons bien ici que l'aimantation orbitale étant due aux courants des électrons magnétiques sur leur orbite, elle est plus localisée autour du noyau que l'aimantation de spin qui est due à la probabilité de présence de ces mêmes électrons magnétiques. En conséquence, dans l'espace réciproque, le facteur de forme orbital décroît moins rapidement que le facteur de forme de spin.

Voyons comme illustration la figure 13 qui montre la carte d'aimantation obtenue par transformée de Fourier inverse pour le composé intermétallique $\mathrm{YCo}_{5}$. Le tableau 2 donne le résultat d'une modélisation en fonctions d'onde où, pour chacun des sites, la partie spin des moments est décomposée en combinaisons caractéristiques de la symétrie du site et où la partie orbitale est traitée de façon isotrope ${ }^{(21)}$.

\subsection{Facteurs de forme des électrons $f$ : terres rares et actinides}

Lorsque le couplage spin orbite est important, le moment angulaire de spin et le moment angulaire orbital se combinent ensemble pour donner un moment angulaire total.

$$
\mathbf{J}=\mathbf{L}+\mathbf{S}
$$

$\mathrm{J}$ est alors un bon nombre quantique $(\mathrm{J}=\mathrm{L}-\mathrm{S}$ pour la première moitié de la série $\mathrm{J}=\mathrm{L}+\mathrm{S}$ pour la deuxième moitié), et on peut écrire le moment magnétique ainsi :

$$
\boldsymbol{\mu}=\mathrm{g}_{\mathrm{J}} \mathbf{J}
$$




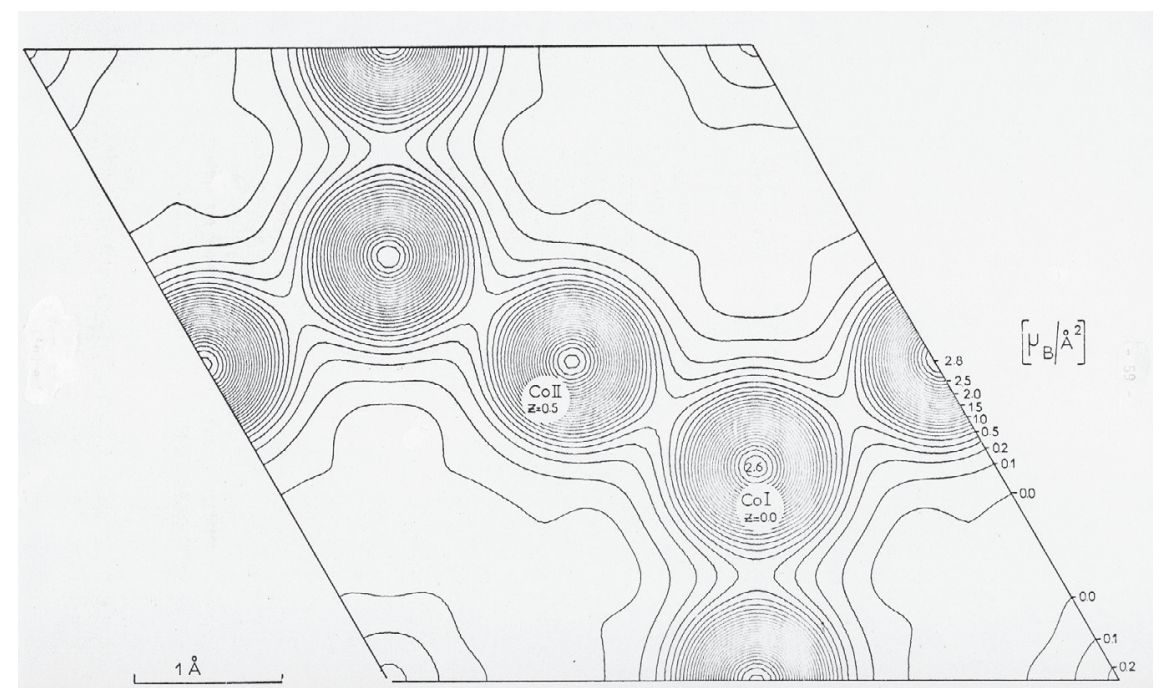

Figure 13. $\mathrm{YCo}_{5}$ : maille élémentaire et densité d'aimantation projetée le long de $\mathbf{c}^{(21)}$.

Tableau 2. Analyse des facteurs de forme des deux sites de cobalt dans $\mathrm{YCo}_{5}\left(\right.$ ref. $\left.^{21}\right)$.

\begin{tabular}{lllll}
\hline Site & Moment localisé & Proportion de spin & Parametres d'occupation \\
\hline $\mathrm{Co}_{\mathrm{I}}$ & $1.77(2) \mu_{\mathrm{B}}$ & $0.74(5)$ & $\mathrm{d}_{z^{2}}$ & $0.23(3)$ \\
& & & $\mathrm{d}_{x z^{\prime}}, \mathrm{d}_{y z}$ & $0.18(12)$ \\
& & $0.84(4)$ & $\mathrm{d}_{x^{2}-y^{2}}, \mathrm{~d}_{x y}$ & 0.58 \\
$\mathrm{Co}_{\mathrm{II}}$ & $1.72(2) \mu_{\mathrm{B}}$ & & $\mathrm{d}_{z^{2}}$ & $0.15(2)$ \\
& & & $\mathrm{d}_{x z}$ & $0.24(4)$ \\
& & $\mathrm{d}_{y z}$ & $0.19(3)$ \\
& & $\mathrm{d}_{x^{2}-y^{2}}$ & $0.22(3)$ \\
Somme des moments localisés dans une maille & $8.90(10) \mu_{\mathrm{B}}$ & & 0.20 \\
Aimantation mesurée pour une maille & $7.99(2) \mu_{\mathrm{B}}$ & \\
\hline
\end{tabular}

Le formalisme complet du facteur de forme atomique est exposé dans ${ }^{(15-17)}$. On trouve les expressions utiles pour ce facteur de forme dans Lander et al. ${ }^{(22)}$.

Le point important concernant les composés de terres rares et d'actinides est la présence de fortes anisotropies dans la densité d'aimantation, un résultat de la forte contribution orbitale pour ces atomes. Ces anisotropies dépendent de la direction du champ appliqué, comme cela est illustré sur la Fig. 14 pour le cérium $^{(23)}$.

L'approximation sphérique donne une expression simplifiée pour le facteur de forme :

$$
f(Q)=\left\langle j_{0}(Q)\right\rangle+C_{2}\left\langle j_{2}(Q)\right\rangle
$$

avec

$$
\mathrm{C}_{2}=\frac{2}{\mathrm{gJ}_{\mathrm{J}}}-1=\frac{\mathrm{J}(\mathrm{J}+1)+\mathrm{L}(\mathrm{L}+1)-\mathrm{S}(\mathrm{S}+1)}{3 \mathrm{~J}(\mathrm{~J}+1)-\mathrm{L}(\mathrm{L}+1)+\mathrm{S}(\mathrm{S}+1)} .
$$

Le Tableau 3 donne les valeurs du coefficient $C_{2}$ pour les différents remplissages de la couche f. On notera le cas particulier d'un remplissage à 5 électrons pour lequel le moment de spin et le moment d'orbite se compensent presque, ce qui donne une allure inhabituelle pour le facteur de forme. Ce cas 

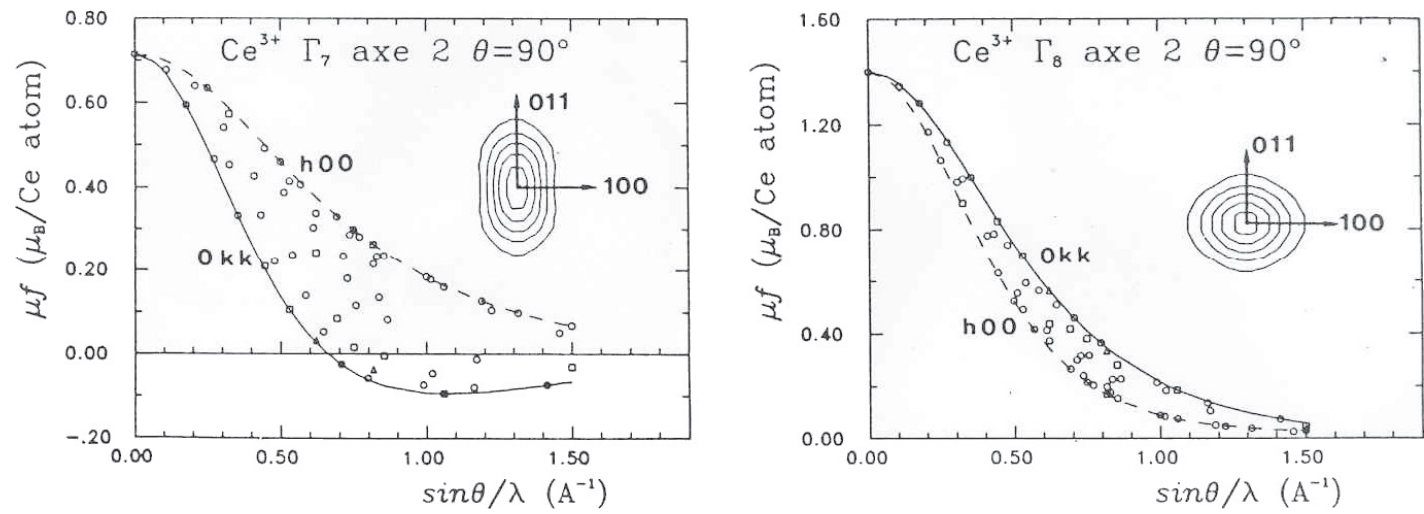

Figure 14. Facteur de forme et densité d'aimantation de $\mathrm{Ce}^{3+}$ calculé pour les deux états cubiques $\Gamma_{7}$ et $\Gamma_{8}^{(23)}$.

Tableau 3. Coefficient $C_{2}$ pour les différents remplissages de la couche $f$.

\begin{tabular}{|c|ccc|c|}
\hline & $\mathrm{L}$ & $\mathrm{S}$ & $\mathrm{J}$ & $\mathrm{C}_{2}=\frac{2}{\mathrm{~g}_{\mathrm{J}}}-1$ \\
\hline $\mathrm{f}^{1}$ & 3 & $1 / 2$ & $5 / 2$ & 1.333 \\
$\mathrm{f}^{2}$ & 5 & 1 & 4 & 1.500 \\
$\mathrm{f}^{3}$ & 6 & $3 / 2$ & $9 / 2$ & 1.750 \\
$\mathrm{f}^{4}$ & 6 & 2 & 4 & 2.333 \\
$\mathrm{f}^{5}$ & 5 & $5 / 2$ & $5 / 2$ & 6.000 \\
$\mathrm{f}^{6}$ & 3 & 3 & 0 & pas de moment \\
$\mathrm{f}^{7}$ & 0 & $7 / 2$ & $7 / 2$ & 0 \\
$\mathrm{f}^{8}$ & 3 & 3 & 6 & 0.333 \\
$\mathrm{f}^{9}$ & 5 & $5 / 2$ & $15 / 2$ & 0.500 \\
$\mathrm{f}^{10}$ & 6 & 2 & 8 & 0.600 \\
$\mathrm{f}^{11}$ & 6 & $3 / 2$ & $15 / 2$ & 0.667 \\
$\mathrm{f}^{12}$ & 5 & 1 & 6 & 0.714 \\
$\mathrm{f}^{13}$ & 3 & $1 / 2$ & $7 / 2$ & 0.750 \\
\hline
\end{tabular}

est illustré sur la Fig. 15 pour $\mathrm{SmCo}_{5}$, où l'existence d'un maximum du facteur de forme à $Q \neq 0$ vient de ce que les localisations dans l'espace ne sont pas les mêmes pour l'aimantation de spin et pour l'aimantation orbitale, les signes de ces deux contributions étant opposées ${ }^{(24)}$.

\section{MODÉLISATION DE LA DENSITÉ DE SPIN : L'EXPANSION MULTIPOLAIRE}

On peut obtenir un modèle plus souple que le précédent en paramétrisant directement la densité de spin (ou la densité d'aimantation) plutôt que de paramétriser la fonction d'onde. Un modèle bien adapté consiste à développer cette densité en multipôles, autour des atomes au repos ${ }^{(24)}$. On a alors une superposition de densités atomiques asphériques, chacune d'entre elles étant décrite par un développement en fonctions harmoniques sphériques réelles $\mathrm{y}_{\ell}^{\mathrm{m}}(\hat{\mathrm{r}})$.

$$
\mathrm{M}(\mathbf{r})=\sum_{\ell=0}^{\infty} \mathrm{R}_{\mathrm{d}}^{\ell}(\mathrm{r}) \sum_{\mathrm{m}=-\ell}^{\ell} \mathrm{P}_{\ell \mathrm{m}} \mathrm{y}_{\ell}^{\mathrm{m}}(\theta, \varphi)
$$

où les $P_{\ell \mathrm{m}}$ sont les coefficients de population et les $\mathrm{R}_{\mathrm{d}}^{\ell}(\mathrm{r})$ sont des fonctions radiales de la densité de spin, par exemple sous la forme de fonctions de Slater. Comme la densité correspond au carré de la fonction d'onde, on peut prendre comme fonctions radiales de la densité $\mathrm{R}_{\mathrm{d}}^{\ell}(\mathrm{r})$ le carré des fonctions 


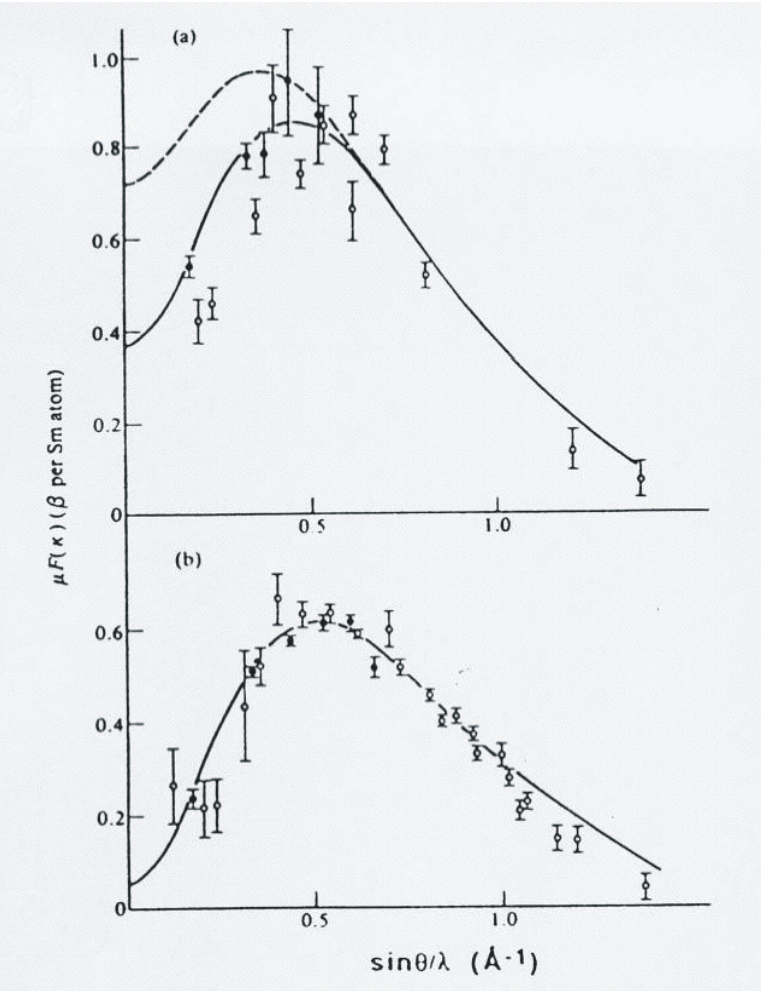

Figure 15. Facteur de forme du Sm mesurée sur le composé $\mathrm{SmCo}_{5}$ à différentes températures ${ }^{(24)}$.

radiales d'amplitude $\mathrm{R}_{\mathrm{a}}^{\ell}(\mathrm{r})$ et les écrire de la façon suivante :

$$
\mathrm{R}_{\mathrm{d}}^{\ell}(\mathrm{r})=\frac{\left.\zeta^{\left(\mathrm{n}_{\mathrm{d}}+3\right.}\right)}{\left(\mathrm{n}_{\mathrm{d}}+2\right) !} \mathrm{r}^{\mathrm{n}_{\mathrm{d}}} \mathrm{e}^{-\zeta \mathrm{r}}
$$

avec $\zeta=2 \alpha$ et $n_{d}=2\left(n_{a}-1\right)$. On a ainsi, pour une orbitale 1 s orbitals $\left(n_{a}=1\right) n_{d}=0$, pour des orbitales $2 \mathrm{~s}$ et $2 \mathrm{p}\left(\mathrm{n}_{\mathrm{a}}=2\right) \mathrm{n}_{\mathrm{d}}=2$, pour des orbitales $3 \mathrm{~s}, 3 \mathrm{p}$ et $3 \mathrm{~d}\left(\mathrm{n}_{\mathrm{a}}=3\right) \mathrm{n}_{\mathrm{d}}=4 \ldots$

Le facteur de structure magnétique s'écrit alors :

$$
\mathrm{F}_{\mathrm{M}}(\mathbf{Q})=\sum_{\text {atoms }}\left\lfloor\sum_{\ell=0}^{\infty} \Phi_{\ell}(\mathrm{Q}) \sum_{\mathrm{m}=-\ell}^{\ell} \mathrm{P}_{\ell \mathrm{m}} \mathrm{y}_{\ell}^{\mathrm{m}}(\hat{\mathrm{Q}})\right\rfloor \mathrm{e}^{\mathrm{i} \mathbf{Q r}} \mathrm{e}^{-\mathrm{W}}
$$

où les intégrales radiales $\Phi_{\ell}(\mathrm{Q})$ sont définies par :

$$
\Phi_{\ell}(\mathrm{Q})=\mathrm{i}^{\ell} \int_{0}^{\infty} \mathrm{R}_{\mathrm{d}}^{\ell}(\mathrm{r}) \mathrm{j}_{\ell}(\mathrm{Qr}) \mathrm{r}^{2} \mathrm{dr}
$$

à l'aide des fonctions de Bessel sphériques $\mathrm{j}_{\ell}(\mathrm{z})$. Les vibrations thermiques sont prises en compte par le terme $\mathrm{e}^{-\mathrm{W}}$. Les fonctions harmoniques sphériques réelles $\mathrm{y}_{\ell}^{\mathrm{m}}(\hat{\mathrm{r}})$ sont des combinaisons linéaires des harmoniques sphériques habituelles $\mathrm{Y}_{\ell}^{\mathrm{m}}(\hat{\mathrm{r}})$ :

$$
\begin{aligned}
& \mathrm{y}_{\ell}^{\mathrm{m}+}=\frac{1}{2}\left(\mathrm{Y}_{\ell}^{\mathrm{m}+}+\mathrm{Y}_{\ell}^{\mathrm{m}-}\right) \\
& \mathrm{y}_{\ell}^{\mathrm{m}-}=\frac{1}{2 \mathrm{i}}\left(\mathrm{Y}_{\ell}^{\mathrm{m}+}+\mathrm{Y}_{\ell}^{\mathrm{m}-}\right) .
\end{aligned}
$$




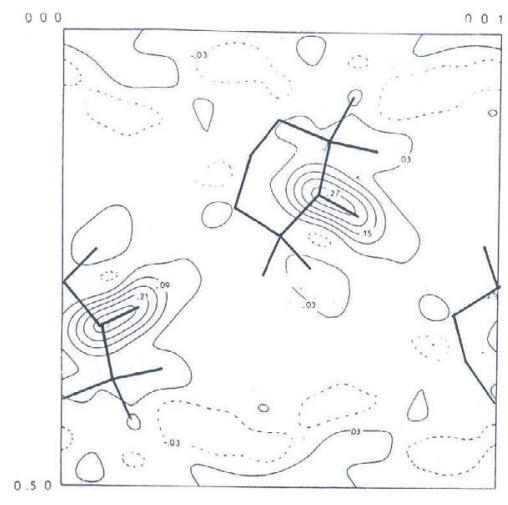

(a)

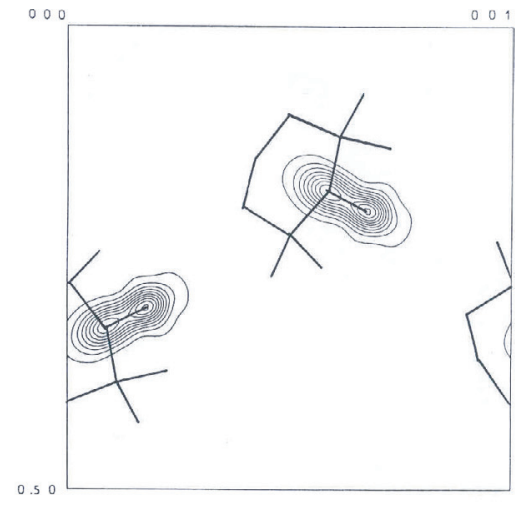

(b)

Figure 16. Suberate de tanol : comparaison entre la distribution de spin reconstituée (a) par inversion de Fourier, et (b) par un développement en multipôles ${ }^{(25)}$.

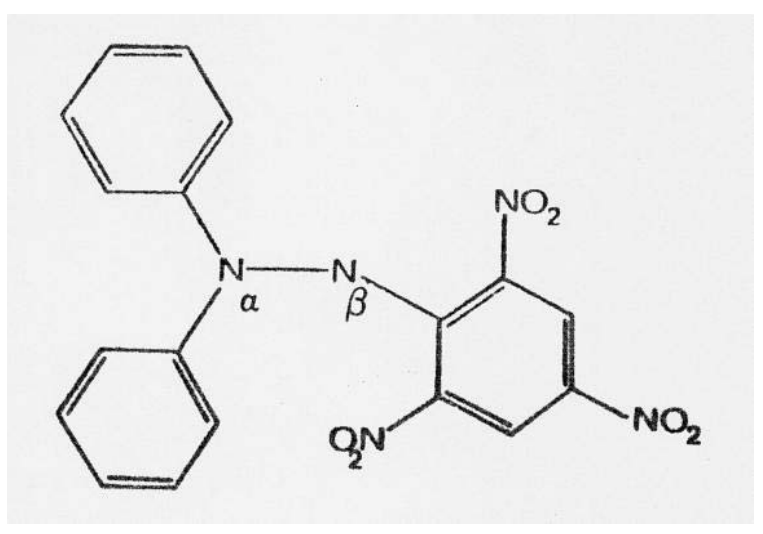

Figure 17. La molecule de diphenyl-picryl-hydrazil (DPPH).

Pour chaque atome, un jeu de paramètres $\zeta, \mathrm{P}_{\ell \mathrm{m}}$ caractérise la distribution de spin. On ajuste ces paramètres par un affinement de moindres carrés des données de diffraction, en général des facteurs de structures magnétiques expérimentaux. Une fois les paramètres obtenus, on peut construire la densité de spin.

Un exemple de cette méthode est donné par la détermination de la densité de spin du subérate de tanol $\left(\mathrm{C}_{13} \mathrm{H}_{23} \mathrm{O}_{2} \mathrm{NO}\right)_{2}$. Cette molécule est un radical libre binitroxyde où les électrons sont localisés sur les deux groupes $\mathrm{NO}$, aux deux extrêmités de la molécule qui a une forme de chaîne. Les rapports de flipping de 69 réflexions de type $(0 \mathrm{kl})$ ont été mesurées, jusqu'à $(\sin \theta / \lambda)_{\max }=0.45 \AA^{-1}$. Ces 69 réflexions mesurées ne sont pas toutes les réflexions contenues dans cette sphère du réseau réciproque; ce sont les plus fortes, c'est à dire celles qui ont les plus grands facteurs de structure nucléaires. A partir de ces mesures on peut reconstruire la projection de la densité de spin le long de $\mathbf{a}^{(25)}$. La Figure 16 compare deux reconstructions de cette densité, obtenues l'une par inversion de Fourier, l'autre par un développement en multipôles. Ce dernier montre moins de bruit et une meilleure résolution.

Cette modélisation de la densité de spin peux s'étendre aux structures cristallines non centrosymétriques. Dans ce cas, l'affinement de moindres carrés compare directement les rapports de flipping expérimentaux aux facteurs de flipping calculés.

La densité de spin du diphenyl-picryl-hydrazil (DPPH) (figure 17) a été déterminée de cette façon ${ }^{(26)}$. Cette molécule cristallise, avec une molécule de solvent $\mathrm{C}_{6} \mathrm{H}_{6}$ dans le groupe d'espace monoclinique 


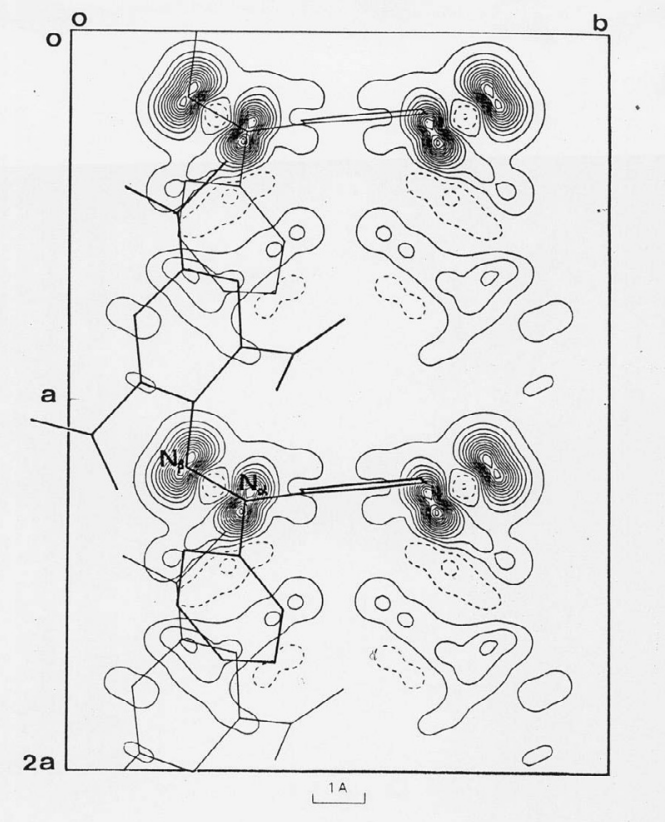

Figure 18. Densité de spin du DPPH reconstituée par un développement multipolaire et projetée le long de l'axe $\mathbf{c}^{(26)}$.

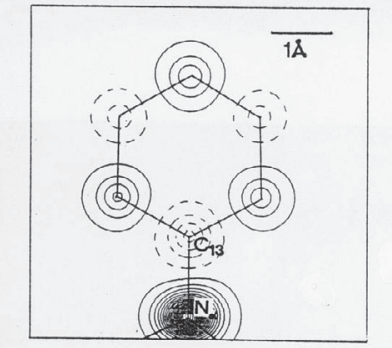

a

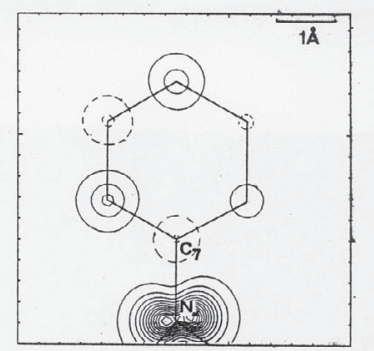

b

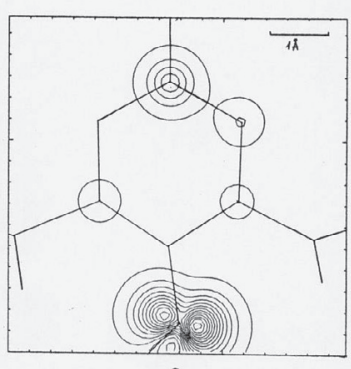

c

Figure 19. Densité de spin du DPPH, obtenue par développement en multipôles et projetées sur les plans des cycles aromatiques. 
Pc qui n'est pas centro-symétrique. La projection selon l'axe c de la densité de spin reconstituée après détermination des paramètres d'un développement multipolaire est représentée sur la Fig. 18.

On voit que la plus grande partie de la densité est localisée et également distribuée sur les deux atomes d'azote de la partie hydrazile, mais qu'une partie de la densité est délocalisée sur les cycles. La Figure 19 represente cette partie délocalisée, projetée sur les plans des cycles. Il faut noter l'alternance positive et négative des signes de la densité sur les atomes de carbone, alternance qui se poursuit sur les atomes $\mathrm{N}$ et $\mathrm{O}$ de l'anneau picryl.

\section{APPENDICE : FONCTIONS ATOMIQUES DE SLATER ET INTÉGRALES RADIALES}

Comme on l'a vu dans le texte, une fonction d'onde atomique à un électron peut être développée en une partie angulaire et une partie radiale :

$$
\varphi(\mathbf{r})=\sum_{\ell=0}^{\infty} \mathrm{R}_{\mathrm{a}}^{\ell}(\mathrm{r}) \sum_{\mathrm{m}=-\ell}^{\ell} \mathrm{a}_{\ell \mathrm{m}} \mathrm{Y}_{\ell}^{\mathrm{m}}(\theta, \varphi)
$$

où $\theta, \varphi$ sont les coordonnées angulaires de $\mathbf{r}$ et où les $\mathrm{Y}_{\ell}^{\mathrm{m}}$ sont les harmoniques sphériques habituelles

On exprime souvent $\mathrm{R}_{\mathrm{a}}^{\ell}(\mathrm{r})$ comme une fonction de Slater :

$$
\mathrm{R}_{\mathrm{a}}^{\ell}(\mathrm{r})=\frac{(2 \alpha)^{\left(\mathrm{n}_{\mathrm{a}}+\frac{1}{2}\right)}}{\sqrt{\left(2 \mathrm{n}_{\mathrm{a}}\right) !}} \mathrm{r}^{\left(n_{a-1}\right)} \mathrm{e}^{-\alpha \mathrm{r}}
$$

Cette fonction dépend explicitement d'un premier nombre quantique $\mathrm{n}_{\mathrm{a}}$ :

$$
\begin{aligned}
& \text { Pour } \mathrm{n}_{\mathrm{a}}=1(1 \mathrm{~s}) \quad \mathrm{R}_{\mathrm{a}}^{\ell}(\mathrm{r})=2 \alpha^{\frac{3}{2}} \mathrm{e}^{-\alpha \mathrm{r}} \\
& \text { Pour } \mathrm{n}_{\mathrm{a}}=2(2 \mathrm{~s}, 2 \mathrm{p}) \quad \mathrm{R}_{\mathrm{a}}^{\ell}(\mathrm{r})=\frac{2 \alpha^{\frac{5}{2}}}{\sqrt{3}} \mathrm{re}^{-\alpha \mathrm{r}} \\
& \text { Pour } \mathrm{n}_{\mathrm{a}}=3(3 \mathrm{~s}, 3 \mathrm{p}, 3 \mathrm{~d}) \quad \mathrm{R}_{\mathrm{a}}^{\ell}(\mathrm{r})=\frac{2^{\frac{3}{2}} \alpha^{\frac{7}{2}}}{3 \sqrt{5}} \mathrm{r}^{2} \mathrm{e}^{-\alpha \mathrm{r}}
\end{aligned}
$$

L'exposant atomique de Slater $\alpha$ est caractéristique de deux nombres quantiques $\mathrm{n}_{\mathrm{a}}$ and $\ell$. Ces exposants ont été tabulés pour les couches électroniques de tous les éléments par Clementi and Roetti ${ }^{(19)}$ (attention : les valeurs sont données en unités atomiques et pas en $\AA^{-1}$ ).

Les intégrales radiales sont les transformées de Bessel-Fourier de ces fonctions radiales :

$$
\left\langle\mathrm{j}_{\mathrm{L}}(\mathrm{Q})\right\rangle=\int_{0}^{\infty} \mathrm{r}^{2}\left(\mathrm{R}_{\mathrm{a}}^{\ell}(\mathrm{r})\right)^{2} \mathrm{j}_{\mathrm{L}}(\mathrm{Qr}) \mathrm{dr} .
$$

où les $\mathrm{j}_{\mathrm{L}}(\mathrm{z})$ sont les fonctions de Bessel sphériques :

$$
\begin{gathered}
\mathrm{j}_{0}(\mathrm{z})=\frac{1}{\mathrm{z}} \sin \mathrm{z} \\
\mathrm{j}_{1}(\mathrm{z})=\frac{1}{\mathrm{z}^{2}} \sin \mathrm{z}-\frac{1}{\mathrm{z}} \cos \mathrm{z} \\
\mathrm{j}_{2}(\mathrm{z})=\left(\frac{3}{\mathrm{z}^{3}}-\frac{1}{\mathrm{z}}\right) \sin \mathrm{z}-\frac{3}{\mathrm{z}^{2}} \cos \mathrm{z} \\
\mathrm{j}_{3}(\mathrm{z})=\left(\frac{15}{\mathrm{z}^{4}}-\frac{6}{\mathrm{z}^{2}}\right) \sin \mathrm{z}+\left(\frac{-15}{\mathrm{z}^{3}}+\frac{1}{\mathrm{z}}\right) \cos \mathrm{z}
\end{gathered}
$$




$$
\mathrm{j}_{4}(\mathrm{z})=\left(\frac{105}{\mathrm{z}^{5}}-\frac{45}{\mathrm{z}^{3}}+\frac{1}{\mathrm{z}}\right) \sin \mathrm{z}+\left(\frac{-105}{\mathrm{z}^{4}}+\frac{10}{\mathrm{z}^{2}}\right) \cos \mathrm{z}
$$

Introduisant ces fonctions dans (31), on obtient :

Pour $\mathrm{n}_{\mathrm{a}}=1(1 \mathrm{~s})$

$$
\left\langle\mathrm{j}_{0}(\mathrm{x})\right\rangle=\frac{1}{\left(1+\mathrm{x}^{2}\right)^{2}}
$$

Pour $\mathrm{n}_{\mathrm{a}}=2(2 \mathrm{~s}, 2 \mathrm{p})$

$$
\left\langle\mathrm{j}_{0}(\mathrm{x})\right\rangle=\frac{1-\mathrm{x}^{2}}{\left(1+\mathrm{x}^{2}\right)^{4}} \quad\left\langle\mathrm{j}_{2}(\mathrm{x})\right\rangle=\frac{2 \mathrm{x}^{2}}{\left(1+\mathrm{x}^{2}\right)^{4}}
$$

Pour $\mathrm{n}_{\mathrm{a}}=3(3 \mathrm{~s}, 3 \mathrm{p}, 3 \mathrm{~d})$

$$
\left\langle\mathrm{j}_{0}(\mathrm{x})\right\rangle=\frac{1-\frac{10}{3} \mathrm{x}^{2}+\mathrm{x}^{4}}{\left(1+\mathrm{x}^{2}\right)^{6}} \quad\left\langle\mathrm{j}_{2}(\mathrm{x})\right\rangle=\frac{\frac{56}{15} \mathrm{x}^{2}-\frac{8}{5} \mathrm{x}^{4}}{\left(1+\mathrm{x}^{2}\right)^{6}} \quad\left\langle\mathrm{j}_{4}(\mathrm{x})\right\rangle=\frac{\frac{16}{3} \mathrm{x}^{4}}{\left(1+\mathrm{x}^{2}\right)^{6}}
$$

$\operatorname{avec} x=\frac{\mathrm{Q}}{2 \alpha(\AA)^{-1}}$.

En tenant compte de ce que $\alpha\left(\AA^{-1}\right)=\alpha($ atomic units $) / \mathrm{a}_{0}$, avec $\mathrm{a}_{0}=0.52918 \AA$,

$$
\mathrm{x}=\frac{\mathrm{Q}}{2 \alpha\left(\AA^{-1}\right)}=\frac{\mathrm{Q}_{0}}{2 \alpha \text { (unités atomiques })}=\frac{2 \pi \mathrm{a}_{0}}{\alpha \text { (unités atomiques })} \frac{\sin \theta}{\lambda} .
$$

\section{Références}

[1] Shull C. G. and Yamada Y., J. Phys. Soc. Jap. 17 Suppl. BIII, 1 (1962).

[2] Boucherle J. X., Givord F., Lejay P., Schweizer J. and Stunault A., Physica B 156-157, 809 (1989).

[3] Zheludev A., Bonnet M., Ressouche E., Schweizer J., Wan M. and Wang H., J. Magn. Magn. Mat. 135, 147 (1994).

[4] James R. W., The Optical Principles of the Diffraction of X rays. The Crystalline State, Vol. 2, Bells and Sons, London, 1954.

[5] Shull C. G. and Mook H. A., Phys. Rev. Lett. 16, 184 (1966).

[6] Mook H. A., Phys. Rev. 148, 495 (1966).

[7] Shannon C., Bell System Tech. J. 27, 623 (1948).

[8] Gull S. F. and Skilling J., IEEE Proceedings 131, 646 (1984).

[9] Papoular R., Gillon B., Europhysics Lett. 13, 429 (1990).

[10] Papoular R., Gillon B., "Neutron Scattering Data Analysis 1990”, edited by Johnson M.W., Adam Hilger Publisher (1990), p. 101.

[11] Gull S. F., Skilling J., Users’ MEMSYS manual, Maximum Entropy Data Consultant Ltd. 1989.

[12] Boucherle J. X., Henry J. Y., Papoular R. J., Rossat-mignod J., Schweizer J., Tasset F. and Uimin G., Physica B 192, 25 (1993).

[13] Papoular R. J. and Delapalme A., Phys. Rev. Lett. 72, 1486 (1994).

[14] Schleger P., Puig-molina A., Ressouche E., Rutty O. and Schweizer J., Acta Cryst. A 53, 426 (1997).

[15] Marshall W. and Lovesey S. W., Theory of Thermal Neutron Scattering (Clarendon press, Oxford, 1971).

[16] Lovesey S.W., Theory of Neutron Scattering from Condensed Matter (Clarendon Press, Oxford, 1983). 
[17] Balcar E. and Lovesey S. W., Theory of Magnetic Neutron and Photon Scattering (Clarendon Press, Oxford, 1989).

[18] Brown J. International Tables for Crystallography, Volume C: Mathematical, Physical and Chemical Tables, edited by A.J.C. Wilson (Kluwer Academic Publishers, 1992), p. 391.

[19] Clementi E., Roetti, Atomic Data and Nuclear Data Tables, Vol 14 (1974), p. 178.

[20] Pontillon Y., Caneschi A., Gatteschi D., Ressouche E., Schweizer J. and Sessoli R. Physica B (1999) (to be published).

[21] Schweizer J. and Tasset F., J. Phys. F. 10, 2799 (1980).

[22] Lander G. H. and Brun T. O., J. Chem. Phys. 53, 1387 (1970).

[23] Boucherle J. X. and Schweizer J., Physica 130, 337 (1985).

[24] Givord D., Laforet J., Schweizer J. and Tasset F., J. Appl. Phys. 50, 2008 (1979).

[25] Brown P. J., Capiomont A., Gillon B. and Schweizer J., J. Mag. Magn. Mat. 14, 289 (1979).

[26] Boucherle J. X., Gillon B., Maruani J. and Schweizer J., Mol. Phys. 60, 1121 (1987). 